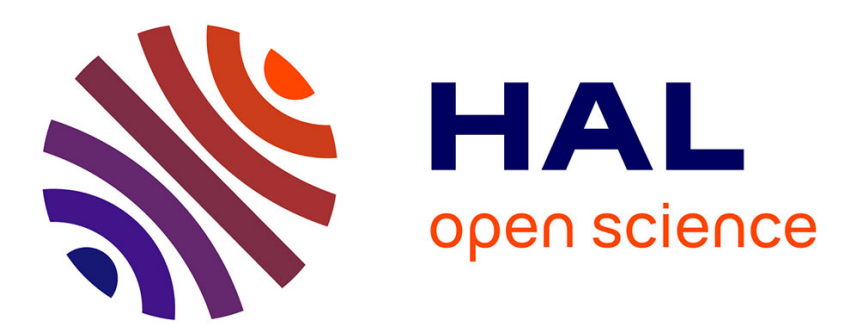

\title{
An accurate modelling of piezoelectric multi-layer plates
}

Amâncio Fernandes, Joël Pouget

\section{To cite this version:}

Amâncio Fernandes, Joël Pouget. An accurate modelling of piezoelectric multi-layer plates. European Journal of Mechanics - A/Solids, 2002, 21 (4), pp.629 - 651. 10.1016/S0997-7538(02)01224-X . hal01802507

\section{HAL Id: hal-01802507 https://hal.sorbonne-universite.fr/hal-01802507}

Submitted on 20 Sep 2019

HAL is a multi-disciplinary open access archive for the deposit and dissemination of scientific research documents, whether they are published or not. The documents may come from teaching and research institutions in France or abroad, or from public or private research centers.
L'archive ouverte pluridisciplinaire HAL, est destinée au dépôt et à la diffusion de documents scientifiques de niveau recherche, publiés ou non, émanant des établissements d'enseignement et de recherche français ou étrangers, des laboratoires publics ou privés. 


\title{
An accurate modelling of piezoelectric multi-layer plates.
}

\author{
Amâncio FERNANDES and Joël POUGET \\ Laboratoire de Modélisation en Mécanique (UMR 7607) \\ Université Pierre et Marie Curie, Case 162, 4 Place Jussieu \\ 75252 Paris Cedex 05, France \\ and \\ Laboratoire d'Etudes Mécaniques des Assemblages (FRE 2481) \\ Université de Versailles/Saint-Quentin-en-Yvelines, 45 Ave des Etats-Unis \\ 78035 Versailles Cedex, France
}

\begin{abstract}
The present work proposes a new approach to laminated plates with piezoelectric layers based on a refinement of the electric potential as function of the thickness coordinate and accounting for shearing correction of the elastic displacement. The approach deals with the combination of an equivalent singlelayer approach for the mechanical displacement with a layerwise-type modelling of the electric potential considered as an additional degree of freedom. Such an approach offers flexibility in accommodating electric conditions at the layer interfaces. The equations governing the force, moment and electric charge resultants of the laminated piezoelectric plate are then deduced from a variational formulation involving mechanical surface loads or prescribed electric potential on the top and bottom faces of the plate as well as at layer interfaces. A particular attention is devoted to the interface conditions which are enforced by using Lagrange multipliers in the variational principle. Emphasis is placed on the performances, advantages and limitations of the present approach. The quality of the predictions of the global and local responses (the through-the-thickness variation of elastic displacements, stresses, electric potential and induction) is quantified for particular structures of practical interest such as piezoelectric bimorph, bilayer structure and piezoelectric sandwich undergoing applied density of force and electric potential. Moreover, comparisons of the results provided by the refined approach to those of finite element computations and simplified model are also presented. The comparisons assess of the effectiveness of the present laminated piezoelectric plate model that improves, in significant way, the predictions given by a simplified approach.
\end{abstract}

Keywords : Piezoelectric composites, laminated plates, higher order plate theory, actuators.

\section{Introduction}

The analysis of piezoelectric composites such as laminated plates requires theories with efficiently accurate approximation of both sensor and actuator functions. The study of novel materials consisting of composite structures equipped with piezoelectric layers remains an active area of research and the success of adaptive devices has attracted the attention of industry due to numerous technological applications (Tani et al., 1998; Sunar and Rao, 1999). The most simple piezoelectric actuator is usually made of single component system (for instance, a slab of piezoelectric ceramics). Typically, such an actuator produces displacements in the order of few micro-meters when applying an electric field up to $1 \mathrm{kV} / \mathrm{mm}$. To overcome this limitation, an actuator using flexural-extensional deformation of thin structures requires several components and it will be a composite material rather than a monolithic structure. One of the most popular multilayer piezoelectric composites commonly used is the piezoelectric bimorph or bender. The application of an electric field across the two layers of the bender causes one layer to expand while the other one shrinks. The global result is a flexural deformation much greater than the length or thickness deformation of the individual layers. This is the actuator or motor function, and 
a magnification of the elastic displacement by a factor of 100 is thus obtained. Conversely, when a piezoelectric bimorph or bi-layer is forced to flex, one layer will be in tension while the other is in compression. The variation of stresses in each layer will produce electric (voltage and current) outputs. Piezoelectric materials and especially piezoelectric composites open completely new possibilities in the design of adaptive or smart structures and very interesting technological applications have been proposed, ranging from aeronautical and automotive structures (shape control of large space antennas, active or passive control of vibrations, etc.) (Rao and Sunar, 1994; Loewy, 1997; Hagood and Von Flotow, 1991; Yoon and Washington, 1998) to miniature positioning devices (micro-robots, medical apparatus, micro-pumps, etc.) (Muralt et al., 1986) and other engineering applications.

It seems, therefore, that a coupled piezoelectric laminated plate approach that can $a c$ curately and efficiently predict the electromechanical state of thin and intermediary thick piezoelectric laminated composites requires a particular attention. The importance of the challenging study of these models was recognized in the literature (Saravanos and Heyliger, 1999; Gopinathan et al., 2000). The present work attempts to develop consistent, yet comprehensive approach to piezoelectric plates made of a stack of piezoelectric or/and non-piezoelectric layers. Quite number of recent studies establishing correct and efficient piezoelectric plate models has been reported (Saravanos and Heyliger, 1999; Wang and Yang, 2000). Here, we propose an approach combining an equivalent single-layer theory for the mechanical displacements including shear effects with a layerwise-type approximation for the electric potential. This approach is also an interesting feature because multilayered piezoelectric structures are appropriate to accommodate multiple voltage actuator inputs and sensor outputs. Furthermore, the present study is a continuation of a paper devoted to single-layered plate (Fernandes and Pouget, 2001-a, 2001-b)

A number of studies attempts to incorporate various representations of approximation through-the-thickness of laminated piezoelectric plates with extensions to shells. Pioneer works on piezoelectric plates were proposed by H.F. Tiersten (Tiersten, 1969), R.D. Mindlin (Mindlin, 1972) and more recently by C.K. Lee (Lee and Moon, 1989; Lee, 1990). Furthermore, various types of piezoelectric laminates are classified according to the kinematic assumptions for approximating the through-the-thickness variation of electromechanical variables, refinement of field approximations and the method of representation of piezoelectric layer. The more common model is based on the elementary beam or plate theories using the kinematic assumption of the Love-Kirchhoff's theory. The simplest approach to piezoelectric beams and plates incorporate effectives forces and moments induced by piezoelectric actuation on plate deformation, however the electric charge equation is not considered in the analysis. This kind of approach is referred usually as induced strain models. The latter models have been presented in the works of B. Wang and C.A. Rogers (Wang and Rogers, 1991), extensions to layerwise approach have been considered by P.F. Pai (Pai et al., 1993) and D.H. Robbins and J.N. Reddy (Robbins and Reddy, 1993).

A large number of formulations assumes that piezoelectric laminate will deform as a single homogeneous layer and the electric charge conservation equation or Gauss law is considered for the sensor function of the laminate. Such an approach, named equivalent single layer model, has been reported mainly by C.K. Lee and F.C. Moon (Lee and Moon, 1989) and C.K. Lee (Lee, 1990). Many authors have considered single-layer theories for the analysis of active and sensor laminated plates and shells, based either on the Love-Kirchhoff's 
assumption or on the first-order shear deformation approximation (Chandrashekhara and Donthireddy, 1997). The approach considers both direct and converse piezoelectric constitutive equations and provides two-dimensional equations of motion for the resultants and effective electric charge equations. The latter equations are however used to compute the electric outputs of the sensor function.

In order to remedy the limitation of the previous approach some attempts have been made to introduce the electric degrees of freedom in addition to the elastic displacement as function of the thickness variable, effective charge equations are then obtained and present the fully piezoelectric coupling effects. This kind of models are usually referred as to coupled single-layer piezoelectric plate theories. Earlier works dealing with coupled single-layered approaches were reported by H.F. Tiersten (Tiersten, 1969) and R.D. Mindlin (Mindlin, 1972). Nevertheless, they did not considered extension to multilayer theories. A number of consistent coupled single-layer piezoelectric plate models were developed by J. Kim et al. (Kim et al., 1997) and J. Wang and co-workers (Wang et al., 1999) leading also to finite element modelling of piezoelectric structures. J.A. Mitchell and J.N. Reddy (Mitchell and Reddy, 1995) propose a hybrid or mixed approach to piezoelectric laminates using the equivalent single-layer assumption for the elastic displacements while the electric potential is considered as discrete-layer approximation providing then excellent predictions of the laminate responses.

Nevertheless, the equivalent single-layer theory turns out to be insufficient in some utmost situations, for rather thick laminates or plates with strong variations of elastic, piezoelectric and dielectric coefficients. Layerwise approaches are therefore considered for which their kinematic and electric potential variations through the plate thickness is smooth enough within each layer and the continuity conditions at the layer interface are also ascertained. Layerwise models incorporate the local electromechanical responses of each layer of the laminate. Lot of works were reported on layerwise approach, among them, we quote the works of E. Carrera (Carrera, 1997), P. Heyliger and D.A. Saravanos (Heyliger and Saravanos, 1995), U. Icardi and M. Di Scuva (Icardi and Di Sciuva, 1996) and J.S. Yang (Yang, 1999). Some of them deal with layerwise approaches mostly dedicated to finite element formulation such as works of D.A. Savaranos et al. (Savaranos et al., 1997) or J. Kim and co-workers (Kim et al., 1997). Extensions to piezoelectric multilayered shells have been proposed by H.S. Tzou (Tzou, 1993) based either on Kirchhoff-Love's hypothesis or on the first-order shear deformation theory.

Another efficient and interesting approach uses asymptotic techniques. The full threedimensional electroelasticity solution is researched for as asymptotic expansions with respect to a small parameter (typically $\varepsilon=h / L$, where $h$ is the plate thickness and $L$ is a characteristic transverse dimension). The solution is then obtained by solving the two-dimensional field equations at successive orders (Maugin and Attou, 1990; Cheng and Batra, 2000; Cheng et al., 2000).

The approach to piezoelectric laminates proposed here includes shear effects approximated by trigonometric functions (Fernandes and Pouget, 2001-a, 2001-b). This particular choice of approximation is motivated by the boundary conditions on the top and bottom faces of the plate that must be satisfied by transverse shear components. In addition, the approach accounts for the fully approximation of the charge equation or Gauss equation. As consequence, we do not consider any hypothesis on the electric displacement or induction. The present consistent approach to piezoelectric laminates is performed in the 
framework of generalized variational formulation for linear piezoelectric materials. The effective equations of motion for the generalized stress and electric charge or induction resultants are then deduced from the variational formulation along with the corresponding boundary conditions. Various situations are considered such as bimorph structure, bilayer piezoelectric plate and sandwich plate undergoing different kinds of electromechanical loads (i) surface density of force applied to the upper face of the plate and (ii) applied electric potential on the top and bottom faces of the plate. Various benchmark tests are then proposed for the cylindrical bending problem of a simply supported piezoelectric plate. With the view of characterizing the performance and capabilities of the present approach but also the limitation of the model some comparisons to finite element computations performed on the 3D problems are presented. Moreover, in order to show the quality of prediction of the plate modelling, the results are compared to those coming from a simplified model based on the Kirchhoff-Love's assumption (no shear effects). One of the key point of the study is to assess the capability of the present approach to describe the global structural response (deflection, elongation, electric potential or charge), as well as the variation of the electromechanical quantities, stresses, electric inductions through the laminated plate thickness for typical slenderness ratios.

The outline of the paper is organized as follows. The formulation of linear piezoelectricity, governing equations and variational principle are briefly stated in Section 2. The field distribution through the thickness is discussed in Section 3, the treatment of the boundary conditions on the plate faces and the interface continuity conditions are also underlined. The governing equations for the plate approach along with the associated mechanical and electric boundary conditions on the plate contour are given in Section 4 . Section 5 is dedicated to the constitutive laws for the generalized forces and moments as well as generalized electric charges. The study of a laminated piezoelectric plate under cylindrical bending is examined in Section 6 for two types of electromechanical load : applied density of force and electric potential. Numerical results and comparisons to finite element method and simplified plate theory and discussions for piezoelectric bimorph, bilayer structure and sandwich plate for different materials and slenderness ratios are proposed in Section 7 . At last, discussion on the most pertinent results, limitations of the model and extensions are presented in Section 8.

\section{Piezoelectricity : variational formulation and governing equations}

In this section we summerize the necessary ingredients about piezoelectricity involved in the following sections. The formulation is based on Hamilton's principle. The advantage of this approach is that it accounts for both mechanical and electric aspects simutaneously. The variational principle can be stated as

$$
\delta \int_{t_{1}}^{t_{2}} \int_{\Omega} \mathcal{L} d v d t+\int_{t_{1}}^{t_{2}} \delta W d t=0
$$

where $\mathcal{L}$ is the density of the Lagrangian functional given by

$$
\mathcal{L}=\frac{1}{2} \rho \dot{u}_{i} \dot{u}_{i}-H\left(S_{i j}, E_{i}\right)
$$

where the first term in the right hand side of Eq.(2) is the kinetic energy density with $u_{i}$ the components of the displacement, $\rho$ is the mass density, $H\left(S_{i j}, E_{i}\right)$ is called the 
electric enthalpy density function with $S_{i j}=u_{(i, j)}=\frac{1}{2}\left(u_{i, j}+u_{j, i}\right)$ the linear part of the strain tensor component and $E_{i}$ the electric field vector. For the linear piezoelectricity the enthalpy density function takes on the form (Maugin, 1985)

$$
H\left(S_{i j}, E_{i}\right)=\frac{1}{2} \sigma_{i j} S_{i j}-\frac{1}{2} D_{i} E_{i}
$$

where $\sigma_{i j}$ are the components of the stress tensor and $D_{i}$ represents the electric displacement or induction vector. The last term in Eq.(1) is the virtual work of the prescribed mechanical and electric quantities on the domain boundary given by

$$
\delta W=\int_{\partial \Omega} T_{i} \delta u_{i} d S+\int_{\partial \Omega} Q \delta \phi d S .
$$

In Eq.(4), $\mathbf{T}$ represents the surface traction and $Q$ is the surface density of electric charge, both applied on the domain boundary $\partial \Omega$. The scalar variable $\phi$ is the electric potential. We restrict ourselves to classical linear piezoelectricity within the framework of the quasi electrostatic approximation which allows for the electric field to be derived from the electric potential as follows

$$
E_{i}=-\phi_{, i}
$$

It is also observed that the piezoelectric material is a perfect isolator and dielectric. On using a classical argument of integration by part, the field equations are (in absence of body force and density of free electric charge)

$$
\left\{\begin{aligned}
\sigma_{i j, j} & =\rho \ddot{u}_{i} \\
D_{i, i} & =0
\end{aligned}\right.
$$

The associated boundary conditions read as

$$
\left\{\begin{array}{llllll}
\sigma_{i j} n_{j}=T_{i} & \text { or } & u_{i} & =\bar{u}_{i} & \text { on } & \partial \Omega, \\
D_{i} n_{i}=Q & \text { or } & \phi & =\bar{\phi} & \text { on } & \partial \Omega .
\end{array}\right.
$$

The above equations must be completed by constitutive laws for $\boldsymbol{\sigma}$ and $\mathbf{D}$. The latter electromechanical quantities are derived from the enthalpy density function

$$
\sigma_{i j}=\frac{\partial H}{\partial S_{i j}}, \quad D_{i}=-\frac{\partial H}{\partial E_{i}} .
$$

For linear piezoelectricity the enthalpy density function usually takes on the following form (Maugin, 1985; Ikeda, 1996)

$$
H\left(S_{i j}, E_{i}\right)=\frac{1}{2} C_{i j p q}^{E} S_{i j} S_{p q}-e_{i p q} E_{i} S_{p q}-\frac{1}{2} \epsilon_{i j}^{S} E_{i} E_{j} .
$$

It has been assumed isothermal processes, moreover the thermomechanical coupling and pyroelectricity effect have been neglected. Consequently, the constitutive equations for linear piezolectricity are

$$
\left\{\begin{aligned}
\sigma_{i j} & =C_{i j p q}^{E} S_{p q}-e_{l i j} E_{l} \\
D_{i} & =e_{i p q} S_{p q}+\epsilon_{i j}^{S} E_{j} .
\end{aligned}\right.
$$

In Eq.(10) $\mathbf{C}^{E}$ is the fourth-order tensor of elasticity coefficients measured in a constant electric field, $\mathbf{e}$ is the third-order tensor of piezoelectricity coefficients and $\boldsymbol{\epsilon}^{S}$ is the secondorder tensor of dielectric coefficients measured in a constant strain. In the following, we 
will focus our attention to materials which possess three mutually perpendicular planes of symmetry (orthotropic symmetry). The matrix form of the constitutive equations (10) is given in Appendix A.

\section{Approximation of the elastic displacement and electric potential}

Along with the accepted kinematic assumptions for the displacement field in most plate theories (Reddy, 1984; Ochoa and Reddy, 1992) we consider an expansion of the displacement as a series of the thickness coordinate. For the present approach, the displacement field and electric potential are assumed to be of the form

$$
\left\{\begin{array}{l}
u_{\alpha}(x, y, z, t)=U_{\alpha}(x, y, t)-z w_{, \alpha}(x, y, t)+f(z) \gamma_{\alpha}(x, y, t), \quad \alpha \in\{1,2\} \\
u_{3}(x, y, z, t)=w(x, y, t) \\
\phi^{(\ell)}(x, y, z, t)=\phi_{0}^{(\ell)}(x, y, t)+z_{\ell} \phi_{1}^{(\ell)}(x, y, t)+P_{\ell}\left(z_{\ell}\right) \phi_{2}^{(\ell)}(x, y, t)+g(z) \phi_{3}^{(\ell)}(x, y, t) .
\end{array}\right.
$$

With $\ell \in\{1, \ldots \ldots, N\}$ is the layer number. In the local expansion (11) $z_{\ell}$ is the thickness coordinate with respect to the mid-plane of the $\ell$ th layer (see Fig.1).

Some comments on the above approximations are in order.

(i) The present approach combines an equivalent single-layer theory for the displacement field with a layerwise-type approximation for the electric potential. The purely elastic plate problem has been extensively examined by M. Touratier (Touratier, 1991). In Eq.(11), $U_{\alpha}$ holds for the middle plane displacement component, $w$ is the deflection and $\gamma_{\alpha}$ represents the shearing function. All the functions are defined at the mid-plane coordinate $(x, y, 0)$.

(ii) For the electric potential (Fernandes, 2000), the first two terms can be associated with the applied electric potential on the plate faces or the continuity condition of the electric potential at the layer interface. The third term is referred as to the induced electric potential by elastic deformation in the $\ell$ th layer. The last term in Eq. $(11)_{3}$ is connected with the shearing deformation. In the present model, the through-the-thickness distribution of the shearing correction is approximated by a trigonometric function. Consequently, we propose the following functions

$$
P_{\ell}\left(z_{\ell}\right)=z_{\ell}^{2}-\left(\frac{h_{\ell}}{2}\right)^{2}, \quad f(z)=\frac{h}{\pi} \sin \left(\frac{\pi z}{h}\right), \quad g(z)=\frac{h}{\pi} \cos \left(\frac{\pi z}{h}\right),
$$

where $h$ is the plate thickness which is supposed to be uniform and $h_{\ell}$ is the thickness of the $\ell$ th layer and $z_{\ell} \in\left[-h_{\ell} / 2, h_{\ell} / 2\right]$.

(iii) We note $z_{I}^{(\ell)}$ the interface coordinate between layers $(\ell)$ and $(\ell+1)$ with respect to the plate mid-plane (see Fig. 1 ). We set $z_{I}^{(N)}=h / 2$ and $z_{I}^{(0)}=-h / 2$ for a plate made of $N$ layers. Therefore we have $z \in\left[z_{I}^{(\ell-1)}, z_{I}^{(\ell)}\right]$. It could be convenient to define the following coordinate change

$$
\left\{\begin{array}{l}
z_{\ell}=z-z_{\ell}^{(0)} \\
z_{\ell}^{(0)}=\frac{1}{2}\left(z_{I}^{(\ell)}+z_{I}^{(\ell-1)}\right)
\end{array}\right.
$$

where $z_{\ell}^{(0)}$ is the coordinate of the $\ell$ th layer mid-plane with respect to that of the plate. 


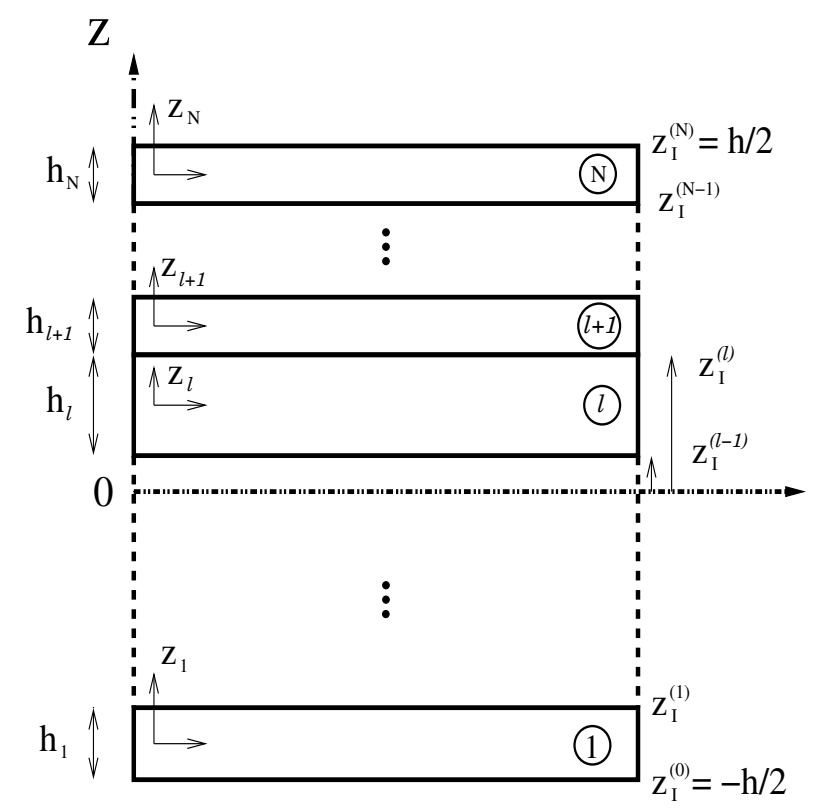

Fig.1 : piezoelectric multi-layered plate.

\section{Boundary and interface conditions}

(i) Two kinds of boundary condition are considered on bottom and top faces of the plate. (a) We consider a force density per unit area applied on the top face of the plate and perpendicular to this face. (b) An electric potential is supposed to be applied on the top and bottom faces of the plate. The latter condition can be written as

$$
\left\{\begin{array}{l}
\phi^{(1)}(x, y,-h / 2, t)=\phi_{0}^{(1)}(x, y, t)-\frac{h_{1}}{2} \phi_{1}^{(1)}(x, y, t)=V^{-}(x, y, t), \\
\phi^{(N)}(x, y,+h / 2, t)=\phi_{0}^{(N)}(x, y, t)+\frac{h_{N}}{2} \phi_{1}^{(N)}(x, y, t)=V^{+}(x, y, t),
\end{array}\right.
$$

thanks to $P_{1}\left(-h_{1} / 2\right)=0, P_{N}\left(+h_{N} / 2\right)=0$ and $g( \pm h / 2)=0$. For the sake of simplicity, we take $V^{+}=V$ and $V^{-}=-V$ and the applied electric potential is supposed to be uniform on the plate faces.

(ii) It is worthwhile discussing the continuity conditions at layer interfaces. Since the layers are perfectly bounded, it is clear that the elastic displacements are continuous at $z=z_{I}^{(\ell)}$. However, the continuity of the electric potential as well as the normal component of the electric induction must be satisfied at $z=z_{I}^{(\ell)}(\ell \in\{1, N-1\})$. Therefore we must write

$$
\left\{\begin{array}{l}
\mathcal{A}_{\ell}=\phi^{(\ell+1)}\left(x, y,-h_{\ell+1} / 2\right)-\phi^{(\ell)}\left(x, y,+h_{\ell} / 2\right)=0 \\
\mathcal{B}_{\ell}=D_{3}^{(\ell+1)}\left(x, y,-h_{\ell+1} / 2\right)-D_{3}^{(\ell)}\left(x, y,+h_{\ell} / 2\right)=0
\end{array}\right.
$$

where the normal component of the electric induction of the $\ell$ th layer is calculated from the constitutive equation $(10)_{2}$

$$
D_{3}^{(\ell)}=e_{3 \alpha \beta}^{*(\ell)} S_{\alpha \beta}-\epsilon_{33}^{*(\ell)} \phi_{, 3}^{(\ell)},
$$

where $e_{3 \alpha \beta}^{*(\ell)}$ and $\epsilon_{33}^{*(\ell)}$ are the effective piezoelectric and dielectric constants of the $\ell$ th layer (see Appendix E). 
(iii) The condition $(15)_{2}$ is accounted for if no electric potential is applied at the interface, otherwise we must replace the condition by the jump condition $\left[D_{3}\right]_{z=z_{I}^{(\ell)}}=Q_{\ell}$ where $Q_{\ell}$ is the surface density of electric charge at the interface $z=z_{I}^{(\ell)}$.

(iv) At this stage of the study, we can use the $2(N-1)$ conditions of continuity (15) to reduce the number of unknown functions and consider the variational principle next. In the present work we consider an other way of solving the problem by introducing the Lagrange multipliers associated with the continuity conditions (15).

(v) Another possible electric boundary condition is electric charges applied on the top and bottom faces of the plate. In this case the boundary condition on the electric induction is $\llbracket \mathbf{D} \rrbracket \cdot \mathbf{n}=Q$, which, in our case, reads as $D_{3}(x, y, z= \pm h / 2, t)=Q(x, y, t)$. In the present work such boundary conditions will not be envisaged.

\section{5 - Equations of the plate model}

The variational formulation stated in Section 2 is then used to derive the equations of the two-dimensional model from the fully three-dimensional theory of piezoelectricity. By substituting the expansions defined in Eq.(11) for the elastic displacement and the electric potential and integrating over the plate thickness we eliminate the dependency of the field on the thickness coordinate $z$. Nevertheless, the continuity conditions (15) are then accounted for by introducing Lagrange multipliers $\mu_{\ell}$ and $\nu_{\ell}$ in the Hamiltonian principle. The variational principle takes on the new following form

$$
\int_{t_{1}}^{t_{2}}\left(\delta K-\delta U+\delta W_{1}+\delta W_{2}+\delta \Lambda\right) d t=0 .
$$

In the present work static processes are only investigated and the kinetic energy is not written down. The second term in Eq.(17) is the variation of the internal force work

$$
\begin{aligned}
\delta U & =\int_{\Sigma}\left\{N_{\alpha \beta}\left(\delta U_{\alpha}\right)_{, \beta}-M_{\alpha \beta}(\delta w)_{, \alpha \beta}+\hat{M}_{\alpha \beta}\left(\delta \gamma_{\alpha}\right)_{, \beta}+\hat{Q}_{\alpha} \delta \gamma_{\alpha}\right. \\
& \left.+\sum_{\ell=1}^{N}\left[\sum_{m=0}^{3} D_{\alpha}^{(m)(\ell)} \delta \phi_{m, \alpha}^{(\ell)}+\sum_{k=1}^{3} D_{3}^{(k)(\ell)} \delta \phi_{k}^{(\ell)}\right]\right\} d S .
\end{aligned}
$$

We introduce the following stress and electric charge or induction resultants

$$
\begin{gathered}
\left(N_{\alpha \beta}, M_{\alpha \beta}, \hat{M}_{\alpha \beta}\right)=\sum_{\ell=1}^{N} \int_{z_{I}^{(\ell-1)}}^{z_{I}^{(\ell)}}(1, z, f(z)) \sigma_{\alpha \beta}^{(\ell)} d z \\
\hat{Q}_{\alpha}=\sum_{\ell=1}^{N} \int_{z_{I}^{(\ell-1)}}^{z_{I}^{(\ell)}} f^{\prime}(z) \sigma_{\alpha 3}^{(\ell)} d z \\
\left(D_{\alpha}^{(0)(\ell)}, D_{\alpha}^{(1)(\ell)}, D_{\alpha}^{(2)(\ell)}, D_{\alpha}^{(3)(\ell)}\right)=\int_{z_{I}^{(\ell-1)}}^{z_{I}^{(\ell)}}\left(1, z-z_{\ell}^{0}, P_{\ell}\left(z-z_{\ell}^{0}\right), g(z)\right) D_{\alpha}^{(\ell)} d z
\end{gathered}
$$




$$
\left(D_{3}^{(1)(\ell)}, D_{3}^{(2)(\ell)}, D_{3}^{(3)(\ell)}\right)=\int_{z_{I}^{(\ell-1)}}^{z_{I}^{(\ell)}}\left(1, P_{\ell}^{\prime}\left(z-z_{\ell}^{0}\right), g^{\prime}(z)\right) D_{3}^{(\ell)} d z
$$

with $P^{\prime}(z)=\frac{d P(z)}{d z}, f^{\prime}(z)=\frac{d f(z)}{d z}$, and $g^{\prime}(z)=\frac{d g(z)}{d z}$. The third and fourth terms in Eq.(17) represent the works of applied forces and electric charges on the plate boundary which are the sum of works of the forces and electric charges applied to the top and bottom faces of the plate and those of the same quantities on the lateral boundary of the plate, namely

$$
\begin{aligned}
\delta W_{1}=\int_{\Sigma}\left\{f_{\alpha} \delta U_{\alpha}-p \delta w+\hat{m}_{\alpha} \delta \gamma_{\alpha}+\sum_{\ell=1}^{N} \sum_{m=0}^{3} q_{m}^{(\ell)} \delta \phi_{m}^{(\ell)}\right\} d S \\
\delta W_{2}=\int_{\mathcal{C}}\left\{F_{\alpha} \delta U_{\alpha}+T \delta w+C_{\alpha} \delta \gamma_{\alpha}-M_{f}(\delta w)_{, n}\right. \\
\left.+\sum_{\ell=1}^{N} \sum_{m=0}^{3} Q_{m}^{(\ell)} \delta \phi_{m}^{(\ell)}\right\} d s-\sum_{p} Z_{p} \delta w_{p} .
\end{aligned}
$$

In Eq.(23), $f_{\alpha}$ and $p$ are the densities of force per unit of area, $\hat{m}_{\alpha}$ is a surface moment density. The generalized electric charges $q_{m}^{(\ell)}$ are given by

$$
\left(q_{0}^{(\ell)}, q_{1}^{(\ell)}, q_{2}^{(\ell)}, q_{3}^{(\ell)}\right)=\left[\left(1, z-z_{\ell}^{0}, P_{\ell}\left(z-z_{\ell}^{0}\right), g(z)\right) D_{3}^{(\ell)}\right]_{z_{I}^{(\ell-1)}}^{z_{I}^{(\ell)}} .
$$

In Eq.(24), $F_{\alpha}$ and $T$ are densities of force per unit of length, $M_{f}$ and $C_{\alpha}$ are lineic moment densities and $Z_{p}$ are transverse forces applied at angular points of the edge boundary contour $\mathcal{C}$ of the plate. Finally, $(\delta w)_{, n}$ is the derivative of the variation $\delta w$ with respect to the normal direction of the boundary contour. The electric charges per unit of length $Q_{m}^{(\ell)}$ are supposed to be zero (no electrode), because the dielectric constant of the piezoelectric material is much larger than that of the outside air.

Finally, the last term in Eq.(17) is the virtual work of forces due to Lagrangian multipliers and it reads as

$$
\delta \Lambda=\sum_{\ell=1}^{N-1} \int_{S_{d}^{(\ell)}} \delta\left[\mu_{\ell} \mathcal{A}_{\ell}+\rho_{e(\ell)}\left(\phi^{e(\ell)}-V^{e(\ell)}\right)+\nu_{\bar{e}(\ell)} \mathcal{B}_{\bar{e}(\ell)}\right] d S,
$$

where $S_{d}^{(\ell)}$ is the surface of the interface between the $\ell$ th and $(\ell+1)$ th layers. In the formulation (26), the case where the electric potential is applied to an interface through a metallic electrode has been considered. In Eq. $(26), e(\ell)$ is the number of the electroded interface for which $\nu_{\bar{e}(\ell)}=0$ and $\bar{e}(\ell)$ is the interface number without electrode for which $\rho_{e(\ell)}=0$. In addition $V^{e(\ell)}$ is the given electric potential on the interface number $e(\ell)$. On taking into account Eq.(15), the constitutive law (16) and approximation (11), straightforward algebra leads to the virtual work of the Lagrangian multipliers

$$
\delta \Lambda=\int_{\Sigma}\left\{A_{\alpha \beta}^{u}\left(\delta U_{\alpha}\right)_{, \beta}-A_{\alpha \beta}^{w}(\delta w)_{, \alpha \beta}+A_{\alpha \beta}^{\gamma}\left(\delta \gamma_{\alpha}\right)_{, \beta}\right.
$$


$\left.+\sum_{\ell=1}^{N-1}\left[\sum_{m=0}^{3}\left(A_{m}^{+(\ell)} \delta \phi_{m}^{(\ell+1)}+A_{m}^{-(\ell)} \delta \phi_{m}^{(\ell)}\right)+\mathcal{A}_{\ell} \delta \mu_{\ell}+\mathcal{B}_{\bar{e}(\ell)} \delta \nu_{\bar{e}(\ell)}+\left(\phi^{e(\ell)}-V^{e(\ell)}\right) \delta \rho_{e(\ell)}\right]\right\} d S$

where the coefficients $A_{\alpha \beta}^{f}$ with $f \in\{u, w, \gamma\}$ depend on the material constants of the layers as well as their thicknesses. The explicit forms of these coefficients are given in Appendix B.

On using integration by part if needed and collecting all the factors of the arbitrary variations $\left\{\delta U_{\alpha}, \delta w, \delta \gamma_{\alpha}, \delta \phi_{m}^{(\ell)}, \delta \mu_{r}, \delta \nu_{\bar{e}(r)}, \delta \rho_{e(r)}\right\}$ with $m \in\{0,1,2,3\}, \ell \in\{1, \cdots, N\}$ and $r \in\{1, \cdots, N-1\}$, we reach the field equations (static process)

$$
\left\{\begin{array}{l}
\mathcal{N}_{\alpha \beta, \beta}+f_{\alpha}=0, \\
\mathcal{M}_{\alpha \beta, \alpha \beta}-p=0, \\
\hat{\mathcal{M}}_{\alpha \beta, \beta}-\hat{Q}_{\alpha}+\hat{m}_{\alpha}=0, \\
D_{\alpha, \alpha}^{(m)(\ell)}-\mathcal{D}_{3}^{(m)(\ell)}+q_{m}^{(\ell)}=0, \quad \ell \in\{1, \cdots, N\} \quad \text { and } m \in\{0,1,2,3\},
\end{array}\right.
$$

where we have introduced the modified force, moment and electric charge resultants

$$
\left\{\begin{array}{l}
\mathcal{N}_{\alpha \beta}=N_{\alpha \beta}-A_{\alpha \beta}^{u}, \\
\mathcal{M}_{\alpha \beta}=M_{\alpha \beta}-A_{\alpha \beta}^{w} \\
\hat{\mathcal{M}}_{\alpha \beta}=\hat{M}_{\alpha \beta}-A_{\alpha \beta}^{\gamma} \\
\mathcal{D}_{3}^{(m)(\ell)}=D_{3}^{(m)(\ell)}-A_{m}^{-(\ell)}-A_{m}^{+(\ell-1)}, \quad \ell \in\{1, \cdots, N\} \quad \text { and } \quad m \in\{0,1,2,3\},
\end{array}\right.
$$

In addition we must set $A_{m}^{+(0)}=A_{m}^{-(N)}=0$ and we have $D_{3}^{(0)(\ell)}=0$. The variation of the Lagrangian multipliers yields the continuity conditions given by Eq.(15) $\left(\mathcal{A}_{\ell}=0\right.$, $\mathcal{B}_{\bar{\eta}(\ell)}=0$ and $\phi^{e(\ell)}-V^{e(\ell)}=0$ on electroded interfaces $\left.z=z_{I}^{e(\ell)}\right)$.

The associated boundary conditions on the plate contour $\mathcal{C}$ are

$$
\left\{\begin{array}{lllll}
F_{\alpha} & =\mathcal{N}_{\alpha \beta} n_{\beta} & \text { or } & U_{\alpha} & \text { given } \\
T & =\left(\tau_{\alpha} \mathcal{M}_{\alpha \beta} n_{\beta}\right)_{, s}+n_{\alpha} \mathcal{M}_{\alpha \beta, \beta} & \text { or } & w & \text { given } \\
M_{f} & =n_{\alpha} \mathcal{M}_{\alpha \beta} n_{\beta} & \text { or } & w_{, n} & \text { given } \\
C_{\alpha} & =\hat{\mathcal{M}}_{\alpha \beta} n_{\beta} & \text { or } & \gamma_{\alpha} & \text { given } \\
D_{\alpha}^{(m)(\ell)} n_{\alpha} & =0 & \text { or } & \phi_{m}^{(\ell)} & \text { given }
\end{array},\right.
$$

with $\ell \in\{1, \cdots, N\}$ and $m \in\{0,1,2,3\}$, where $s$ is the curvilinear coordinate along the contour $\mathcal{C}$. Moreover, we have $\left[\tau_{\alpha} M_{\alpha \beta} n_{\beta}-Z_{p}\right]_{A_{p}}=0$ at the angular points $A_{p}$ of the plate contour where $\boldsymbol{\tau}$ is the tangent vector to the contour $\mathcal{C}$.

Comments : (i) The first two Eq.(28) are similar to those of the Love-Kirchhoff first-order theory of elastic thin plates, the third equation governs the shearing effects.

(ii) The set of equations $(28)_{4}$ are deduced from the conservation law of the electric charge or the Gauss equation. It is worthwhile noticing the presence, in $\operatorname{Eq}(28)_{4}$, of the variation of surface density of electric charge $q_{m}^{(\ell)}$ between the interfaces of the $\ell$ th layer.

(iii) For an applied electric potential on the top and bottom faces of the plate, the conditions given by Eq.(14) must be considered. Consequently, the variation of the unknown 
functions $\phi_{0}^{(1)}$ and $\phi_{0}^{(N)}$ are no longer arbitrary, then Eqs.(28) ${ }_{4}$ for $m=0$ and $\ell=1$ or $N$ disappear.

\section{6 - Constitutive relations for multilayered piezoelectric plates.}

We consider, here, the constitutive laws for the linear piezoelectricity as stated by Eq.(10) or (A.1). On using the stress and electric induction resultants defined by Eqs(19)-(22) and integrating over the plate thickness we are able to put the constitutive equations for the generalized resultants in the following matrix form

$$
\left[\begin{array}{c}
\mathbf{N} \\
\mathbf{M} \\
\hat{\mathbf{M}} \\
\mathbf{D}_{3}
\end{array}\right]=\left[\begin{array}{cccc}
\mathbb{A} & \mathbb{B} & \mathbb{E} & \mathbb{P}_{1} \\
\mathbb{B} & \mathbb{D} & \hat{\mathbb{E}} & \mathbb{P}_{2} \\
\mathbb{E} & \hat{\mathbb{E}} & \hat{\mathbb{D}} & \mathbb{P}_{3} \\
\mathbb{P}_{1}^{T} & \mathbb{P}_{2}^{T} & \mathbb{P}_{3}^{T} & \mathbb{H}
\end{array}\right]\left[\begin{array}{c}
\mathbf{S}^{(0)} \\
\mathbf{S}^{(1)} \\
\mathbf{S}^{(2)} \\
\mathbf{F}_{1}
\end{array}\right]
$$

where the block matrices are given in Appendix $\mathrm{C}$ and we have set the following vectors (we use the Voigt notation for sake of convenience)

$$
\mathbf{N}=\left[\begin{array}{l}
N_{1} \\
N_{2} \\
N_{6}
\end{array}\right], \quad \mathbf{M}=\left[\begin{array}{c}
M_{1} \\
M_{2} \\
M_{6}
\end{array}\right], \quad \hat{\mathbf{M}}=\left[\begin{array}{c}
\hat{M}_{1} \\
\hat{M}_{2} \\
\hat{M}_{6}
\end{array}\right]
$$

for the in-plane resultant, the moment resultant and moment resultant produced by shearing effect, respectively,

$$
\mathbf{S}^{(j)}=\left[\begin{array}{c}
S_{1}^{(j)} \\
S_{2}^{(j)} \\
S_{6}^{(j)}
\end{array}\right]
$$

for the generalized strains defined by

$$
S_{\alpha \beta}^{(0)}=U_{(\alpha, \beta)}, \quad S_{\alpha \beta}^{(1)}=-w_{, \alpha \beta}, \quad S_{\alpha \beta}^{(2)}=\gamma_{(\alpha, \beta)},
$$

with $\alpha, \beta \in\{1,2\}$ and the parentheses mean the symmetric part of the tensor. In addition $\mathbf{D}_{3}$ and $\mathbf{F}_{1}$ are vectors of $3 N$ dimension given by

$$
\mathbf{D}_{3}=\left[D_{3}^{(1)(1)}, D_{3}^{(2)(1)}, D_{3}^{(3)(1)}, \cdots, D_{3}^{(1)(N)}, D_{3}^{(2)(N)}, D_{3}^{(3)(N)}\right]^{T},
$$

and

$$
\mathbf{F}_{1}=\left[\phi_{1}^{(1)}, \phi_{2}^{(1)}, \phi_{3}^{(1)}, \cdots, \phi_{1}^{(N)}, \phi_{2}^{(N)}, \phi_{3}^{(N)}\right]^{T} .
$$

The second constitutive law has also the matrix form

$$
\left[\begin{array}{c}
\hat{\mathbf{Q}} \\
\mathbf{D}_{1} \\
\mathbf{D}_{2}
\end{array}\right]=\left[\begin{array}{ccc}
\mathbb{R} & \mathbb{L}_{1} & \mathbb{L}_{2} \\
\mathbb{L}_{1}^{T} & \mathbb{T}_{1} & 0 \\
\mathbb{L}_{2}^{T} & 0 & \mathbb{T}_{2}
\end{array}\right]\left[\begin{array}{c}
\mathbf{G} \\
\mathbf{F}_{0,1} \\
\mathbf{F}_{0,2}
\end{array}\right],
$$


with the vectors

$$
\mathbf{G}=\left[\begin{array}{c}
\gamma_{1} \\
\gamma_{2}
\end{array}\right], \quad \hat{\mathbf{Q}}=\left[\begin{array}{c}
\hat{Q}_{1} \\
\hat{Q}_{2}
\end{array}\right]
$$

and

$$
\begin{gathered}
\mathbf{D}_{\alpha}=\left[D_{\alpha}^{(0)(1)}, D_{\alpha}^{(1)(1)}, D_{\alpha}^{(2)(1)}, D_{\alpha}^{(3)(1)}, \cdots, D_{\alpha}^{(0)(N)}, D_{\alpha}^{(1)(N)}, D_{\alpha}^{(2)(N)}, D_{\alpha}^{(3)(N)}\right]^{T} \\
\mathbf{F}_{0}=\left[\phi_{0}^{(1)}, \phi_{1}^{(1)}, \phi_{2}^{(1)}, \phi_{3}^{(1)}, \cdots, \phi_{0}^{(N)}, \phi_{1}^{(N)}, \phi_{2}^{(N)}, \phi_{3}^{(N)}\right]^{T}
\end{gathered}
$$

with $\alpha \in\{1,2\}$ and $\mathbf{F}_{0, \alpha}$ holds for the derivative with respect to $x_{\alpha}$. All the block matrices involved in Eqs(31) and (37) are explicitly defined in Appendix C. The components of the matrices depend on the layer geometry (layer thickness and interface coordinates) and material constants (elasticity, piezoelectricity and dielectric permittivity) and they are defined in Appendix D. Some components of the above block matrices can be zero due to their particular form and the zero material constants.

Remarks: In the case of an electric potential applied to the bottom and top faces of the plate, the unknown functions $\phi_{0}^{(1)}$ and $\phi_{0}^{(N)}$ depend on the functions $\phi_{1}^{(1)}$ and $\phi_{1}^{(N)}$ and also on $V^{ \pm}$through Eq.(14). Accordingly, the dimension of the vector $\mathbf{F}_{0, \alpha}$ is reduced to $4 N-2$. Moreover, the corresponding equations (see Eq.(28) $)_{4}$ ) for $\ell=1, m=0$ and $\ell=N, m=0$ disappear. The electric potential $V^{ \pm}$is then considered as an applied field in the plate equations.

\section{7 - Laminated piezoelectric plate under cylindrical bending.}

One of most interesting situations is the study of the laminated piezoelectric plate undergoing an applied surface force density or/and electric potential under cylindrical bending, because the equations of piezoelectric plate can be easily solved by means of Fourier series. Moreover, this situation can be compared to finite element method. It is assumed no shear traction $\left(f_{\alpha}=0\right)$ and no surface moment density $\left(\hat{m}_{\alpha}=0\right)$ on the plate faces. The simple support conditions for a rectangular plate of length $L$ and width $l$ are given by

$$
\left\{\begin{array}{l}
\sigma_{11}(0, z)=\sigma_{11}(L, z)=0 \\
\sigma_{13}(0, z)=\sigma_{13}(L, z)=0 \\
u_{3}(0, z)=u_{3}(L, z)=0 .
\end{array}\right.
$$

It is worthwhile noting that the boundary conditions along the contour $\mathcal{C}$ given by Eq.(30) are obviously satisfied for the cylindrical bending configuration.

In addition, all stresses, strains, displacements, electric field and potential do not depend on the $y$ variable, so that the displacement $u_{2}$ plays no role in the study. As consequence, we set $U_{2}=0$ and $\gamma_{2}=0$. The electromechanical load functions can be expressed in the form of Fourier series as follows

$$
(p(x), V(x))=\sum_{n=1}^{\infty}\left(S_{n}, V_{n}\right) \sin \left(\lambda_{n} x\right),
$$


with $\lambda_{n}=n \pi / L$ and

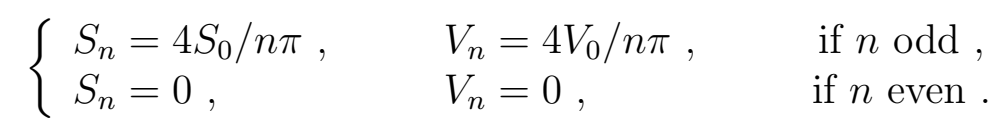

The loads defined by Eqs(42-43) represent uniform applied surface density of force $S_{0}$ and electric potential $V_{0}$. A solution to $\operatorname{Eqs}(28)$ along with the constitutive equations (31)(40) which satisfies the boundary conditions of the cylindrical bending of a plate simply supported takes on the form

$$
\begin{gathered}
\left(U_{1}(x), \gamma_{1}(x)\right)=\sum_{n=1}^{\infty}\left(U_{n}, \Gamma_{n}\right) \cos \left(\lambda_{n} x\right), \\
\left(w(x), \phi_{0}^{(\ell)}(x), \phi_{1}^{(\ell)}(x), \phi_{2}^{(\ell)}(x), \phi_{3}^{(\ell)}(x)\right)=\sum_{n=1}^{\infty}\left(W_{n}, \Phi_{0, n}^{(\ell)}, \Phi_{1, n}^{(\ell)}, \Phi_{2, n}^{(\ell)}, \Phi_{3, n}^{(\ell)}\right) \sin \left(\lambda_{n} x\right),
\end{gathered}
$$

and the same type of series holds for the Lagrange multipliers

$$
\left(\mu_{r}, \nu_{\bar{e}(r)}, \rho_{e(r)}\right)=\sum_{n=1}^{\infty}\left(M_{n}^{(r)}, N_{n}^{\bar{e}(r)}, R_{n}^{e(r)}\right) \sin \left(\lambda_{n} x\right) \quad r \in\{1, N-1\} .
$$

The Fourier coefficients in the above series are determined by substituting the solution (44)-(46) into the plate equations and constitutive equations, next solving simultaneously a set of linear algebraic equations for each $n$ where the right hand side contains the electromechanical loads given by Eq.(42). The set of linear algebraic equations for the Fourier coefficients can be taken on the form

$$
\mathbb{A}_{n} \mathbf{X}_{n}=\mathbf{B}_{n}
$$

where $\mathbb{A}_{n}$ is a square matrix of $6 N+1$ order in the case of the open circuit configuration and of $6 N-1$ order in closed-circuit conditions or applied electric potential. The vector $\mathbf{X}_{n}$ contains the Fourier coefficients

$$
\mathbf{X}_{n}=\left\{U_{n}, W_{n}, \Gamma_{n}, \Phi_{m, n}^{(\ell)}, M_{n}^{(r)}, N_{n}^{\bar{e}(r)}, R_{n}^{e(r)}\right\} \quad m \in\{0,1,2,3\}, \ell \in\{1, N\}, r \in\{1, N-1\} .
$$

The vector $\mathbf{B}_{n}$ contains the applied fields as function of the Fourier factors $S_{n}$ and $V_{n}$. The matrix $\mathbb{A}_{n}$ and vector $\mathbf{B}_{n}$ are function of $\lambda_{n}$, the layer geometry and material constants. 


\section{8 - Numerical results and comparisons.}

This section attempts to quantitavely demonstrate the performance of the present approach to piezoelectric laminated plates. Numerical simulations are performed for typical configurations and they are mostly used as benchmark tests. In addition, the results are directly compared with those provided by finite element formulation and with solutions extracted from a simplified theory (based on kinematic assumptions of Love-Kirchhoff's theory keeping however quadratic terms in the expansion of the electric potential) and they are presented in order to place the advantages and limitations of the refined approach in evidence. Additional comparisons were considered to exact 3D solution to laminated plates which are merely extensions of the Pagano's work to piezoelectric laminated plates (Heyliger and Brook, 1996; Bisegna and Maceri, 1996; Fernandes, 2000). Nevertheless, there is no noticeable discrepancies between the results coming from the finite element computations and those given by the exact solutions (Fernandes, 2000). Two mechanical load configurations are considered corresponding to practical applications : (i) sensor function with a force density per unit area applied to the upper face and (ii) actuator function with an electric potential applied to the top and bottom faces of the plate. Attention will be focussed on particular structures undergoing cylindrical bending among them (i) piezoelectric bimorph, (ii) bi-layer structure made of a piezoelectric layer glued to a non-piezoelectric but elastic layer and (iii) piezoelectric sandwich plate consisting of composite layer coated with two piezoelectric layers including the intermediary electroded configuration. The capability of the present approach is characterized by illustrating the local variation of electromechanical variables through-the-thickness of the plate and global response such as deflection of the plate, the maximum of the induced electric potential and stresses at layer interfaces. The material properties of the layers used for the numerical simulations are given in Tables 1 and 2 .

\begin{tabular}{l|cccccccccc}
\hline \hline & $\begin{array}{c}C_{11}^{E} \\
(G P a)\end{array}$ & $C_{12}^{E}$ & $C_{33}^{E}$ & $C_{13}^{E}$ & $C_{44}^{E}$ & $\begin{array}{c}e_{31} \\
\left(C / m^{2}\right)\end{array}$ & $e_{33}$ & $e_{15}$ & $\begin{array}{c}\epsilon_{11}^{*} \\
(n F / m)\end{array}$ & $\epsilon_{33}^{*}$ \\
\hline PZT-4 & 139. & 77.8 & 115. & 74.3 & 25.6 & -5.2 & 15.1 & 12.7 & 13.06 & 11.51 \\
\hline \hline
\end{tabular}

Table 1: Independent elastic, piezoelectric and dielectric constants of piezoelectric materials (transversally isotropic symmetry).

The geometry of the plate is $L=25 \mathrm{~mm}$ and $l=12.5 \mathrm{~mm}$ and different slenderness ratios are considered $L / h=5,10$ and 50 depending on the situation to be examined. Furthermore, the numerical results for the electromechanical variables are given with the following dimensionless units

(i) for a density of force per unit area $S_{0} \neq 0\left(S_{0}=1000 \mathrm{~N} / \mathrm{m}^{2}\right)$ we set

$$
(U, W, \Phi)=\frac{C_{00}^{E}}{h S_{0}}\left(u_{1}, u_{3}, \phi / E_{0}\right), \quad\left(T_{i j}, \mathcal{D}_{k}\right)=\frac{1}{S_{0}}\left(\sigma_{i j}, E_{0} D_{k}\right)
$$

(ii) for an applied electric potential $V_{0} \neq 0$ ( $V_{0}=50$ volts) we have

$$
(U, W, \Phi)=\frac{E_{0}}{V_{0}}\left(u_{1}, u_{3}, \phi / E_{0}\right), \quad\left(T_{i j}, \mathcal{D}_{k}\right)=\frac{h E_{0}}{C_{00}^{E} V_{0}}\left(\sigma_{i j}, E_{0} D_{k}\right),
$$

for numerical convenience we take $E_{0}=10^{10}$ volts $/ \mathrm{m}$ and $C_{00}$ is a reference elastic constant depending on the situation to be examined. The number of terms in the series Eqs(42)-(46) 
are adjusted according to the slenderness ratios, electromechanical loads and selection of materials thus considered in order to ascertain the series convergence. The finite element computations for comparison are carried out with ABAQUS code by using plane strain elements of 8-node biquadratic type and 800 elements are considered.

\begin{tabular}{c|cccccccccc}
\hline \hline & $\begin{array}{c}C_{11}^{E} \\
(G P a)\end{array}$ & $C_{12}^{E}$ & $C_{22}^{E}$ & $C_{23}^{E}$ & $C_{55}^{E}$ & $\begin{array}{c}e_{31} \\
\left(C / m^{2}\right)\end{array}$ & $e_{33}$ & $e_{15}$ & $\begin{array}{c}\epsilon_{11}^{*} \\
(n F / m)\end{array}$ & $\epsilon_{33}^{*}$ \\
\hline Composite & 134.86 & 5.1563 & 14.352 & 7.1329 & 5.654 & 0 & 0 & 0 & 0.031 & 0.0266 \\
\hline \hline
\end{tabular}

Table 2: Independent elastic, piezoelectric and dielectric constants of a composite made of graphite fibers along $x$ direction in epoxy matrix, $C_{13}^{E}=C_{12}^{E}, C_{22}^{E}=C_{11}^{E}, C_{66}^{E}=C_{55}^{E}$ and $C_{44}^{E}=$ $\frac{1}{2}\left(C_{22}^{E}-C_{23}^{E}\right), \epsilon_{22}^{S}=\epsilon_{33}^{S}$.

\section{1 - Piezoelectric bimorph}

In this situation both piezoelectric layers are made of identical materials and have the same thickness $0.5 h$, however, the piezo-active axes are in opposite directions (see Fig.2). We take $C_{00}=C_{11}^{E}(P Z T-4)$. Such a piezoelectric structure is an excellent candidate to design versatile actuators and micro-positioning devices (Muralt et al., 1986; Smits et al., 1991). In this problem, two Lagrangian multipliers are only necessary in order to enforce the continuity conditions on the electric potential and normal component of the electric induction. The matrix $\mathbb{A}_{n}$ associated with the set of linear equations is a $11 \times 11$ matrix.

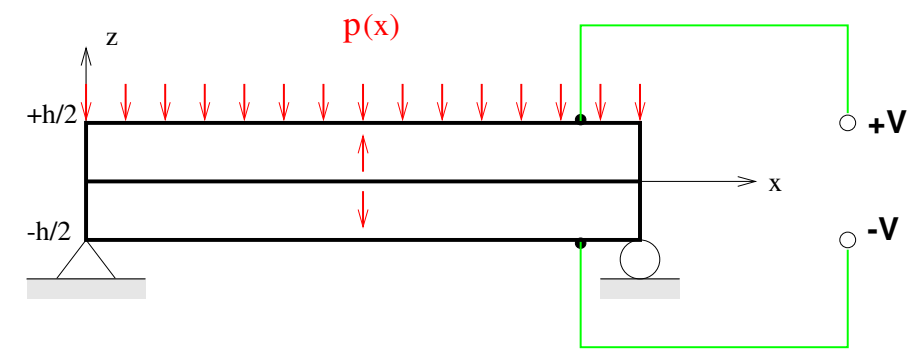

Fig.2 : Piezoelectric bimorph setting.

a - Surface density force

The numerical results are collected in Fig. 3 in dimensionless units. In a first case, the piezoelectric bimorph, made of two PZT-4 layers, suffers an applied force density normal to the top face with the electric potential specified to be zero $(V=0)$. The discrepancy between the maximum values of the deflection, at the plate center, for the present approach and finite element computation for the full 3D model is given in Table 3. A comparison to the simplified model (no shearing correction) is also presented in Table 3 for three typical aspect ratios $(L / h=5,10$ and 50$)$. The induced electric potential at $x=L / 2$ displaying an asymmetric profile is plotted in Fig.3.a. The normal shear stress $\sigma_{13}$ computed at $x=L / 4$ is given in Fig.3.b. It is worthwhile noting the continuity of the normal shear through the interface between piezoelectric layers while it is identically zero for the elementary approach based on the kinematic hypothesis of Love-Kirchhoff. Table 3 tells us that the discrepancy for the deflection between the present approach and finite element results is about $0.01 \%$ for $L / h=50,0.07 \%$ for $L / h=10$ up to $2.5 \%$ for $L / h=5$ (thick plate) while it is over $14 \%$ for the simplified model. The estimate of the 
maximum value of the electric potential is also excellent, it is about $0.007 \%$ for $L / h=50$.
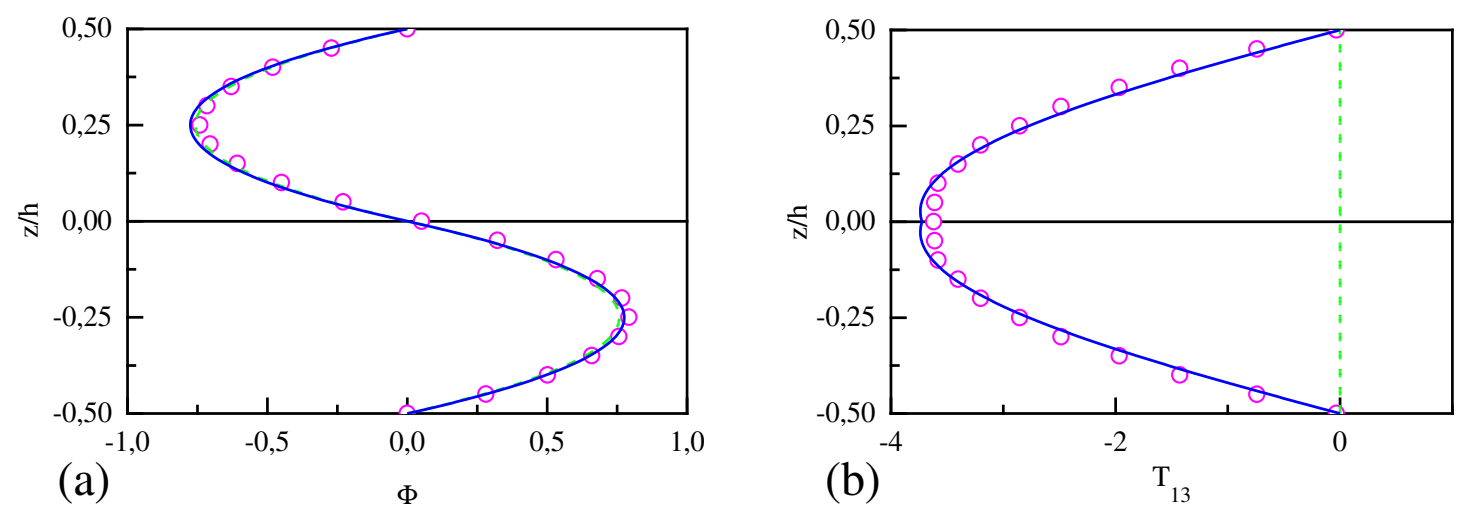

Fig.3 : Force density applied on the top face of a piezoelectric bimorph in closed circuit for $L / h=10$. Plate model (full line), finite element (small circles) and simplified plate model (dashed-line).

\begin{tabular}{cccccccc}
\hline \hline L/h & Approaches & $W$ & Error & $\Phi$ & Error & $T_{13}$ & Error \\
& & $(L / 2,0)$ & $\%$ & $(L / 2,-h / 4)$ & $\%$ & $(L / 4,0)$ & $\%$ \\
\hline \multirow{3}{*}{50} & F.E.M. & $-1.4286 \times 10^{6}$ & & 19.0 & & -18.31 & \\
& Present & $-1.4287 \times 10^{6}$ & 0.007 & 18.986 & 0.007 & -18.625 & 1.7 \\
& L.K. & $-1.4268 \times 10^{6}$ & 0.12 & 18.986 & 0.007 & 0.0 & 100.0 \\
\hline \multirow{2}{*}{10} & F.E.M. & -2360.0 & & 0.7925 & & -3.62 & \\
& Present & -2358.36 & 0.07 & 0.775 & 2.15 & -3.726 & 2.9 \\
& L.K. & -2283.18 & 3.25 & 0.7574 & 4.43 & 0.0 & 100.0 \\
\hline \multirow{2}{*}{5} & F.E.M. & -165.7 & & 0.2234 & & -1.80 & \\
& Present & -161.468 & 2.55 & 0.2063 & 7.64 & -1.86 & 5.67 \\
& L.K. & -142.74 & 13.85 & 0.188 & 15.7 & 0.0 & 100.0 \\
\hline \hline
\end{tabular}

Table 3: Piezoelectric bimorph, applied density force.

\section{b - Applied electric potential}

This situation concerns a piezoelectric bimorph subject to an electric potential applied to the top and bottom faces of the structure $(-V$ at $z=-h / 2$ and $+V$ at $z=+h / 2$ with $p=0)$. The deflection at the plate center is given in Table 4 in comparison to those provided by the finite element computations and the simplified model. The error does not overtake $2.3 \%$ for $L / h=5,10$ and 50 . For $L / h=50$ with an applied electric potential of the order of 100 Volts, the bender produces a deflection of the order of $30 \mu \mathrm{m}$. The deflection variations through-the-thickness at the plate center is presented in Figs 4.a. At last, Fig.4.b shows an interesting result for the component $\mathcal{D}_{3}$ of the electric induction which is almost constant through the plate thickness. Table 4 also exhibits the errors in estimating the jump of the stress $T_{11}$ at the interface and the electric charge at the plate faces between the three approaches according to the slenderness ratios. This shows the efficiency of our refined model to predict both local states (profiles) and global responses for the actuator function of the piezoelectric bimorph. 

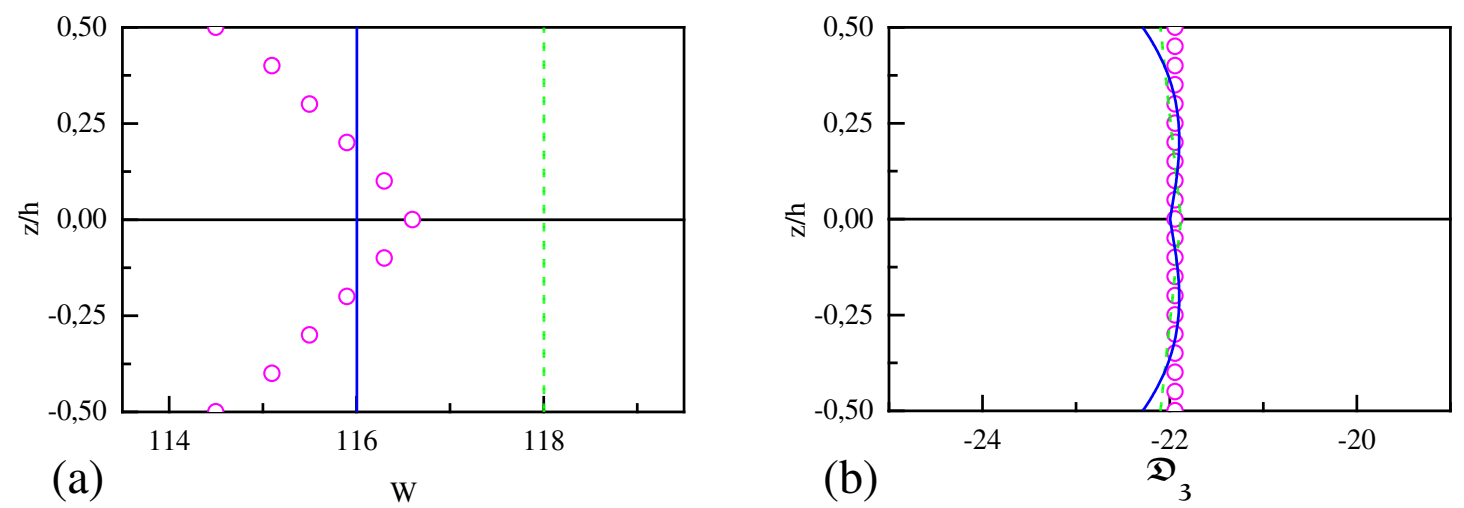

Fig.4 : Electric potential applied to a piezoelectric bimorph for $L / h=10$.

\begin{tabular}{cccccccc}
\hline \hline L/h & Approaches & $\begin{array}{c}W \\
(L / 2,0)\end{array}$ & $\begin{array}{c}\text { Error } \\
\%\end{array}$ & $\begin{array}{c}\llbracket T_{11} \rrbracket \\
(L / 2,0)\end{array}$ & $\begin{array}{c}\text { Error } \\
\%\end{array}$ & $\begin{array}{c}\mathcal{D}_{3} \\
(L / 2, h / 2)\end{array}$ & $\begin{array}{c}\text { Error } \\
\%\end{array}$ \\
\hline \multirow{3}{*}{50} & F.E.M. & 2945.0 & & 2.431 & & -21.94 & \\
& Present & 2943.3 & 0.02 & 2.422 & 0.36 & -21.97 & 0.15 \\
& L.K. & 2945.3 & 0.05 & 2.413 & 0.74 & -21.89 & 0.22 \\
\hline \multirow{2}{*}{10} & F.E.M. & 116.6 & & 2.43 & & -21.94 & \\
& Present & 116. & 0.5 & 2.425 & 0.26 & -21.995 & 0.25 \\
& L.K. & 118. & 1.2 & 2.412 & 0.78 & -21.88 & 0.27 \\
\hline \multirow{2}{*}{5} & F.E.M. & 28.296 & & 2.43 & & -21.94 & \\
& Present & 27.653 & 2.3 & 2.425 & 0.26 & -21.99 & 0.25 \\
& L.K. & 29.651 & 4.8 & 2.412 & 0.78 & -21.88 & 0.27 \\
\hline \hline
\end{tabular}

Table 4: Electric potential applied to a piezoelectric bimorph.

\section{2 - Piezolectric bi-layer.}

Now, we consider a more complex situation than that of the bimorph when both layers are made of different materials and have different thicknesses. The lower layer is made of composite material, more precisely graphite fibers in an epoxy matrix with $h_{1}=0.8 h$ while the upper layer is an PZT-4 piezoelectric ceramics with $h_{2}=0.2 h$. The composite is non-piezoelectric but elastic and dielectric. The material coefficients are given in Tables 1 and 2 and we have $C_{00}=C_{11}^{E}$ (Composite). As in the previous case we present the responses of the structure suffering two electromechanical loads. The electric potential is applied to the piezoelectric layer using metallic electrodes at the interface $\left(z=z_{I}\right)$ and at the top face of the plate $(z=h / 2)$. A sketch of the bi-layer configuration is depicted in Fig.5. Then, the boundary conditions on the electric potential must be examined in details, those are

$$
\left\{\begin{array}{l}
\phi^{(1)}\left(z=+z_{I}\right)=\phi^{(2)}\left(z=+z_{I}\right)=-V \\
\phi^{(2)}(z=+h / 2)=+V
\end{array}\right.
$$

with $z_{I}=\left(h_{1}-h_{2}\right) / 2$. There is no electric potential imposed on the bottom face of the 
lower layer (no electrode), nevertheless, it is assumed there is no density of electric charge, too. Then the boundary condition reads as

$$
D_{3}^{(1)}(z=-h / 2)=0 .
$$

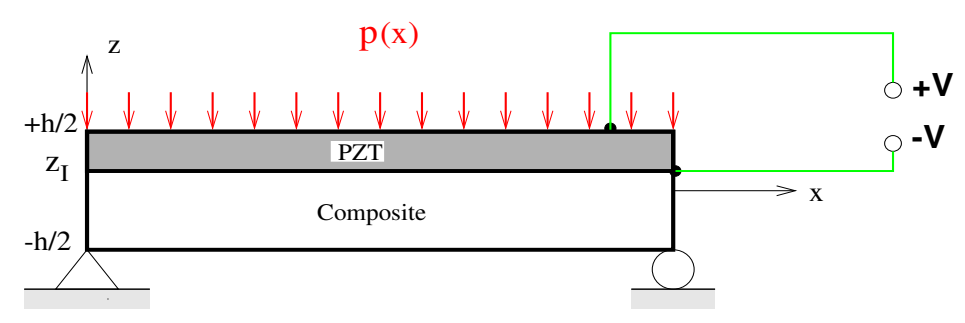

Fig.5 : Piezoelectric bi-layered plate setting.

It is obvious that the continuity of the electric potential at the layer interface is ascertained. As a consequence, there is no continuity condition on the normal component of the electric induction and the Lagrangian multipliers associated with the equations of continuity are not considered. On using the conditions Eq.(47) the electric potential within each layer takes on the form

$$
\left\{\begin{array}{l}
\phi^{(1)}=\phi_{0}^{(1)}+z_{1} \phi_{1}^{(1)}+P_{1}\left(z_{1}\right) \phi_{2}^{(1)}-\frac{g(z)}{g\left(z_{1}\right)}\left(V+\phi_{0}^{(1)}+\frac{h_{1}}{2} \phi_{1}^{(1)}\right), \\
\phi^{(2)}=\phi_{0}^{(2)}+2 \frac{z_{2}}{h_{2}}\left(V-\phi_{0}^{(2)}\right)+P_{2}\left(z_{2}\right) \phi_{2}^{(2)}-2 \frac{g(z)}{g\left(z_{I}\right)} \phi_{0}^{(2)}
\end{array}\right.
$$

with

$$
\begin{gathered}
z_{1}=z+h_{2} / 2, \quad z_{2}=z-h_{1} / 2, \\
P_{1}\left(z_{1}\right)=\left(z-z_{I}\right)(z+h / 2), \quad P_{2}\left(z_{2}\right)=\left(z-z_{I}\right)(z-h / 2),
\end{gathered}
$$

and $g(z)$ is the cosine function defined by Eq.(12). The number of unknown functions needed to solve the problem completely is now 8, namely, $\left\{U_{1}, w, \gamma_{1}, \phi_{0}^{(1)}, \phi_{1}^{(1)}, \phi_{2}^{(1)}, \phi_{0}^{(2)}, \phi_{2}^{(2)}\right\}$. Regarding to the boundary condition Eq.(48), the latter is used for computing the generalized electric charges in Eq.(28) 4 defined by Eq.(25). However, a density of electric charge does exist on the layer interface at $z=z_{I}$. This density is then given by $\llbracket D_{3} \rrbracket_{z=z_{I}}=Q$. It should be noticed knowing that the lower layer is not piezolectric, therefore $D_{3}^{(1)}=-\epsilon_{33}^{*(1)} \phi_{3,3}^{(1)}$ where the electric potential is given by Eq. $(49)_{1}$. a - Applied surface density of force $(V=0)$.

The numerical results and comparisons are shown in Fig. 6 for the aspect ratio $L / h=10$. The elongational displacement, deflection, induced potential and longitudinal stress are plotted in Figs.6.a, 6.b, 6.c and 6.d, respectively. We observe that the through-thethickness distribution of the present plate model is very close to the results given by finite element computations. Conversely, the prediction of the deflection and the induced electric potential given by the simplified model is less accurate. Some characteristic values of electromechanical variables such as the maximum of the deflection at the plate center, the extremum of the electric potential within the piezoelectric layer and the normal component of the electric induction at the upper face are presented in Table 5 for the three slenderness ratios. We note the excellent results for the present model and the errors are less than $0.3 \%$ for $L / h=50$. 


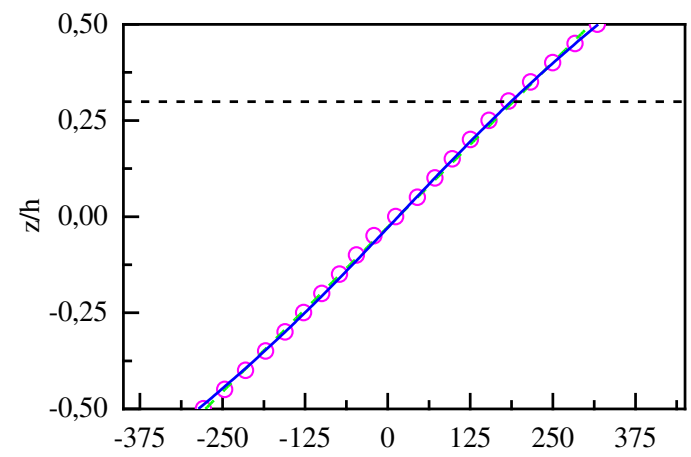

(a)

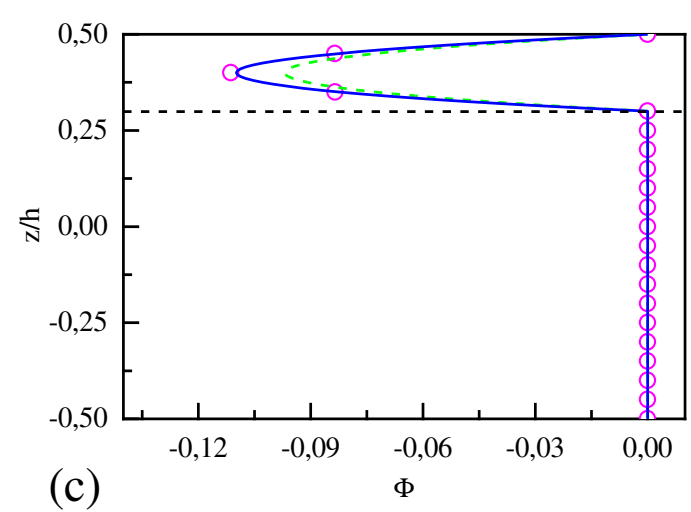

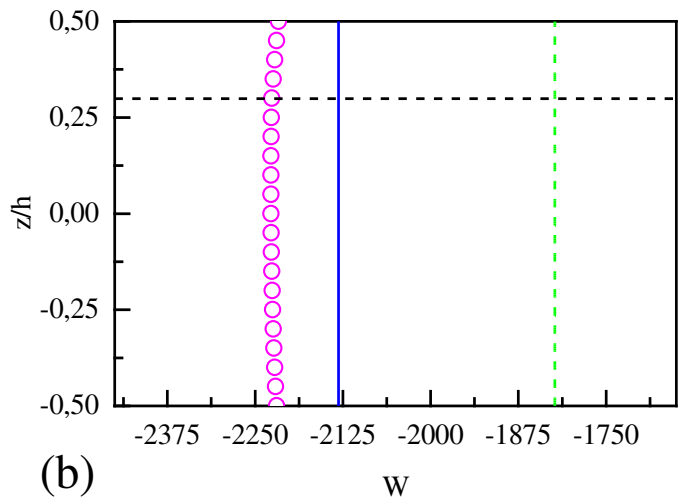

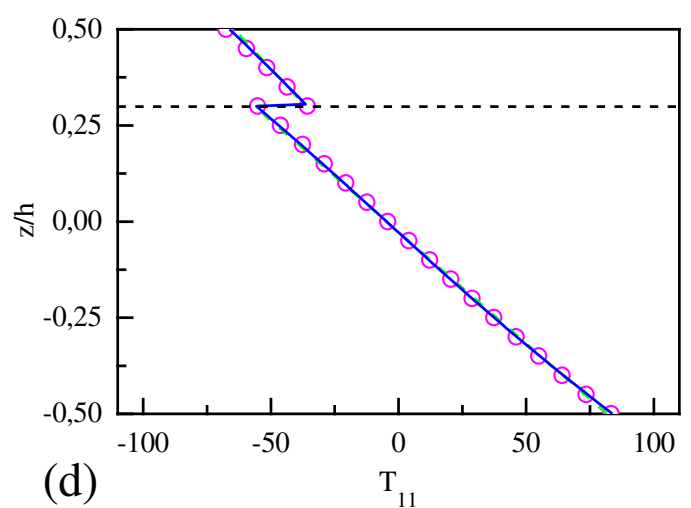

Fig.6 : Force density applied on the top face of a piezoelectric bi-layer in closed circuit for $L / h=10$.

\begin{tabular}{cccccccc}
\hline \hline L/h & Approaches & $\begin{array}{c}W \\
(L / 2,0)\end{array}$ & $\begin{array}{c}\text { Error } \\
\%\end{array}$ & $\begin{array}{c}\Phi \\
\left(L / 2,\left(h_{1}-h_{2}\right) / 2\right)\end{array}$ & $\begin{array}{c}\text { Error } \\
\%\end{array}$ & $\begin{array}{c}\mathcal{D}_{3} \\
(L / 2, h / 2)\end{array}$ & $\begin{array}{c}\text { Error } \\
\%\end{array}$ \\
\hline \multirow{3}{*}{50} & F.E.M. & $-1.15 \times 10^{6}$ & & -2.4392 & & 2070.0 & \\
& Present & $-1.147 \times 10^{6}$ & 0.25 & -2.4377 & 0.06 & 2071.7 & 0.082 \\
& L.K. & $-1.139 \times 10^{6}$ & 0.92 & -2.4249 & 0.58 & 2071.8 & 0.087 \\
\hline \multirow{2}{*}{10} & F.E.M. & -2227.19 & & -0.1113 & & 82.241 & \\
& Present & -2131.22 & 4.3 & -0.1098 & 1.3 & 82.781 & 0.65 \\
& L.K. & -1823.086 & 18.1 & -0.09696 & 12.8 & 82.877 & 0.77 \\
\hline \multirow{2}{*}{5} & F.E.M. & -234.697 & & -0.0384 & & 20.328 & \\
& Present & -190.442 & 18.8 & -0.0371 & 3.38 & 20.628 & 2.8 \\
& L.K. & -113.943 & 51.4 & -0.0242 & 37 & 20.724 & 3.3 \\
\hline \hline
\end{tabular}

Table 5: Piezoelectric bi-layer, applied density force.

b - Applied electric potential ( $p=0)$.

The interesting situation is an electric potential applied to the piezolectric layer. Here, when an electric potential is applied to the active piezoelectric layer, the elongation or contraction of the piezoelectric layer is hindered by the non-responsive but elastic layer and the bi-layer composite suffers a bending motion. Figure 7.a shows the elongational deformation with a quasi-linear variation through the plate thickness. Moreover, we ob- 


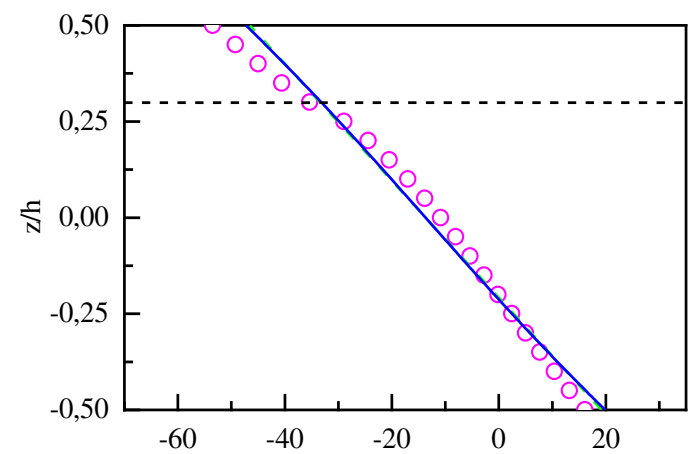

(a)

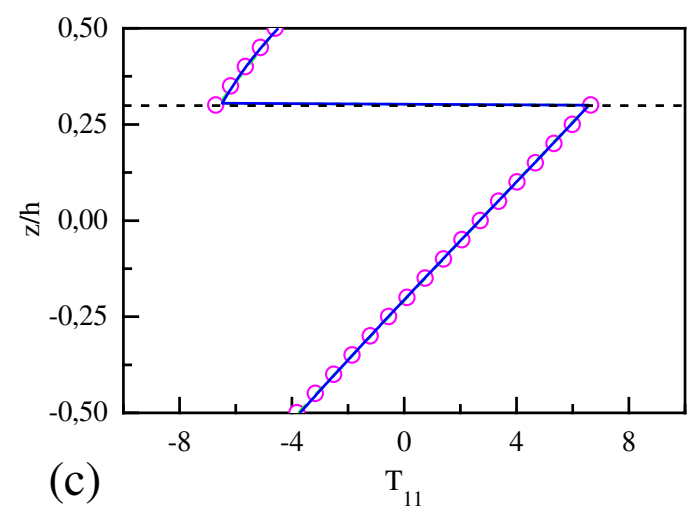

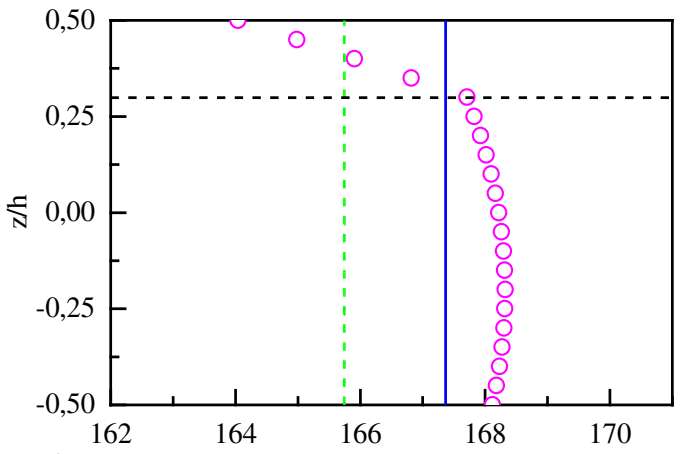

(b)
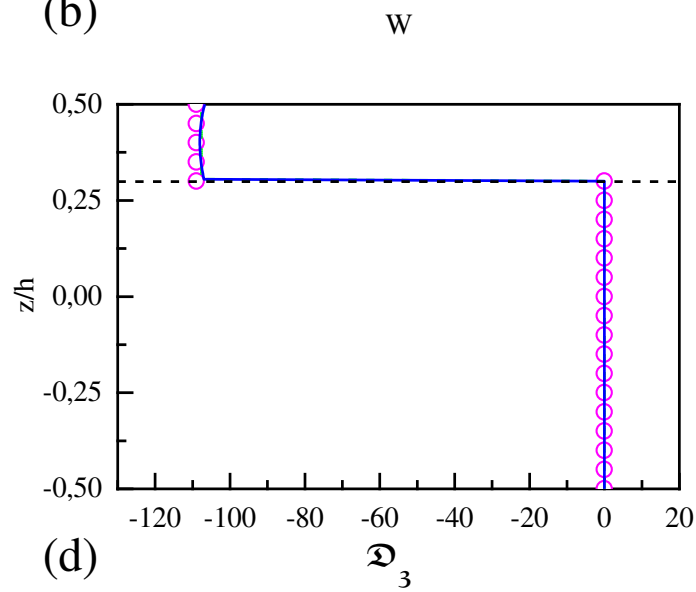

Fig.7 : Electric potential applied to piezoelectric bi-layer for $L / h=10$.

serve that the piezoelectric bi-layer is subject to a deflection as depicted in Fig.7.b. The elongational stress and the normal electric induction are shown in Fig.7.c and Fig.7.d, respectively. A particularly interesting result is that, in this case, we do not have a pure deflection of the plate, in fact, it is accompanied by a small global elongation of the plate as depicted in Fig.7.a $(U(x=0, z=0) \neq 0)$. Our laminated plate model provides a good prediction for both local and global responses in comparison to the results coming from

\begin{tabular}{cccccc}
\hline \hline L/h & Approaches & $\begin{array}{c}W \\
(L / 2,0)\end{array}$ & $\begin{array}{c}\text { Error } \\
\%\end{array}$ & $\begin{array}{c}U \\
(0,0)\end{array}$ & $\begin{array}{c}\text { Error } \\
\%\end{array}$ \\
\hline \multirow{3}{*}{50} & F.E.M. & 4146.25 & & -241.222 & \\
& Present & 4145.197 & 0.025 & -233.155 & 3.3 \\
& L.K. & 4143.585 & 0.064 & -232.869 & 3.5 \\
\hline \multirow{2}{*}{10} & F.E.M. & 168.22 & & -53.4888 & \\
& Present & 167.367 & 0.5 & -47.2719 & 11.6 \\
& L.K. & 165.743 & 1.5 & -46.5741 & 13.0 \\
\hline \multirow{2}{*}{5} & F.E.M. & 43.8665 & & -29.9969 & \\
& Present & 43.0617 & 1.83 & -24.0863 & 19.7 \\
& L.K. & 41.4359 & 5.54 & -23.2873 & 22.4 \\
\hline \hline
\end{tabular}

Table 6: Piezoelectric bi-layer, applied electric potential. 
the simplified model. The errors are presented in Table 6 for the three characteristic slenderness ratios. The results agree very well with those predicted by the finite element computations. The discrepancy for the deflection is about $0.025 \%$ for $L / h=50,0.5 \%$ for $L / h=10$ and it is less than $2 \%$ for a rather thick plate.

\section{3 - Piezoelectric sandwich plate.}

In this part we consider a three-layered plate made of a composite layer (graphite fibres in an epoxy matrix) sandwiched with two piezoelectric layers (PZT-4 ceramics. It has been set $C_{00}=C_{11}^{E}$ (Composite). ). The top and bottom faces of both piezoelectric layers are recovered with conducting electrodes as depicted in Fig.8. The boundary conditions for the electric potentials for the layers are

$$
\left\{\begin{array}{l}
\phi^{(1)}(z=-h / 2)=+V, \\
\phi^{(1)}\left(z=-z_{I}\right)=\phi^{(2)}\left(z=-z_{I}\right)=-V, \\
\phi^{(2)}\left(z=+z_{I}\right)=\phi^{(3)}\left(z=+z_{I}\right)=-V, \\
\phi^{(3)}(z=+h / 2)=+V,
\end{array}\right.
$$

with, in the present case, $z_{I}=h_{2} / 2$. The continuity conditions on the electric potential at the interfaces are obviously ascertained. Moreover, there is no continuity condition on the normal electric induction component and the Lagrangian multipliers therefore disappear.

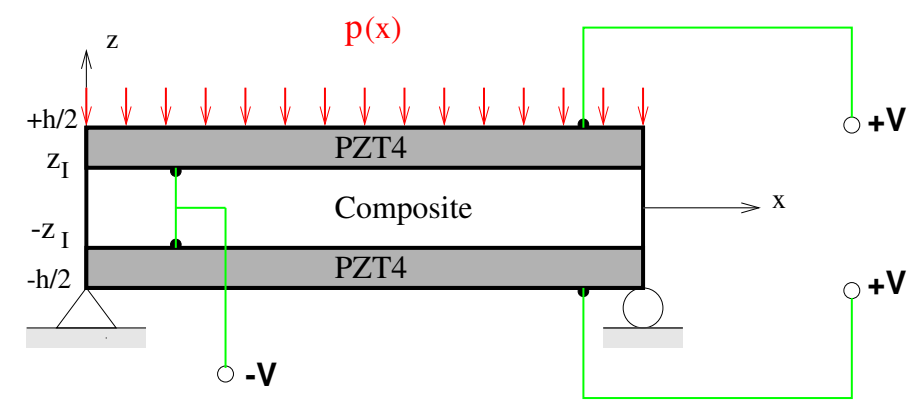

Fig.8 : Piezoelectric sandwich plate with intermediate electrodes.

Owing that the middle layer is a non piezoelectric material the fields $\phi_{2}^{(2)}$ and $\phi_{3}^{(2)}$ are dropped out. Accounting for the boundary and continuity conditions Eq.(50), the electric potential for the three layers can be written as

$$
\left\{\begin{array}{l}
\phi^{(1)}=\phi_{0}^{(1)}+2 \frac{z_{1}}{h_{1}}\left(\phi_{0}^{(1)}-V\right)+P_{1}\left(z_{1}\right) \phi_{2}^{(1)}-2 \frac{g(z)}{g\left(z_{I}\right)} \phi_{0}^{(1)} \\
\phi^{(2)}=-V \\
\phi^{(3)}=\phi_{0}^{(3)}-2 \frac{z_{3}}{h_{3}}\left(\phi_{0}^{(3)}-V\right)+P_{3}\left(z_{3}\right) \phi_{2}^{(3)}-2 \frac{g(z)}{g\left(z_{I}\right)} \phi_{0}^{(3)}
\end{array}\right.
$$

with

$$
\begin{gathered}
z_{1}=z+\frac{1}{2}\left(h_{1}+h_{2}\right), \quad z_{3}=z-\frac{1}{2}\left(h_{1}+h_{2}\right), \quad z_{I}=h_{2} / 2 \\
P_{1}\left(z_{1}\right)=\left(z+z_{I}\right)(z+h / 2), \quad P_{3}\left(z_{3}\right)=\left(z-z_{I}\right)(z-h / 2),
\end{gathered}
$$

and $g(z)$ is still given by Eq.(12). The number of unknown functions is mostly reduced and the problem involved finally 7 unknown functions, namely $\left\{U_{1}, w, \gamma_{1}, \phi_{0}^{(1)}, \phi_{2}^{(1)}, \phi_{0}^{(3)}, \phi_{2}^{(3)}\right\}$. 
Two kinds of electromechanical loads are considered for the numerical simulations as in the previous cases.

a - Surface density of force.

In this case the applied electric potential is set to zero (closed circuit, $V=0$ ) and a density of force per area is applied to the top face of the sandwich plate. From Fig.9, we see the variation of the longitudinal displacement, deflection, induced electric potential and electric induction $\mathcal{D}_{3}$ as function of the thickness coordinate. It turns out, once more, that the refined model including shearing correction improves the prediction in a significant way. We note the difference with the simplified model especially for the deflection. The error is clearly increased when the slenderness ratio $L / h$ is getting smaller as shown in Table 7 . The interesting picture is the profile of the electric potential, the latter does not have linear variation it is, instead, piecewise parabolic within the piezoelectric layers. The higher order terms in the expansion Eq.(51) 3 play a crucial role in the prediction of the local response. For $L / h=50$ the maximum value of the induced electric potential is about -5.2 Volts. The profile plotted in Fig.9.d shows very distinctly the jump of $\mathcal{D}_{3}$ at the layer interface. This means that a surface density of electric charge is produced on the interfaces and on the top and bottom faces of the sandwich plate. The electric charge thus produced on the interface electrodes is of the order of $1.1 \times 10^{-2} \mathrm{C} / \mathrm{m}^{2}$.
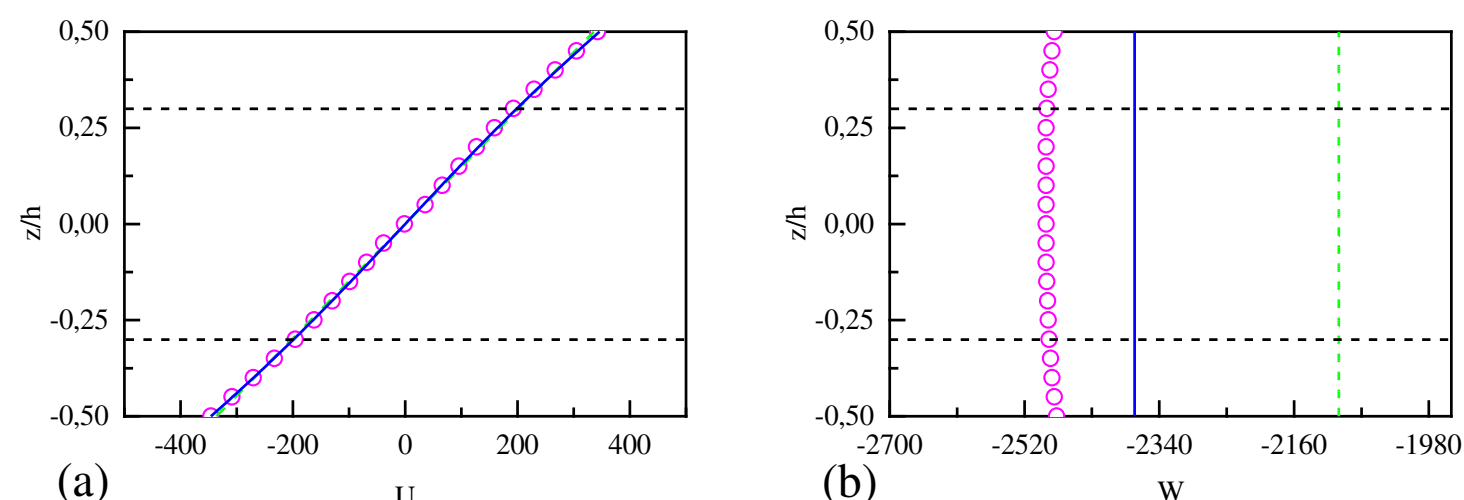

(b)

W

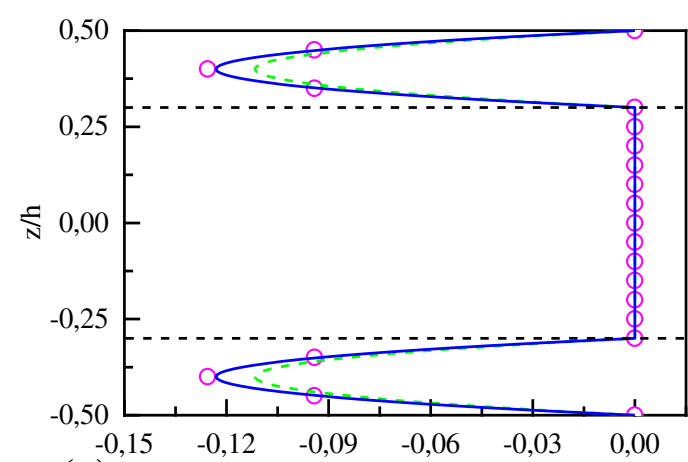

(c)

$\Phi$

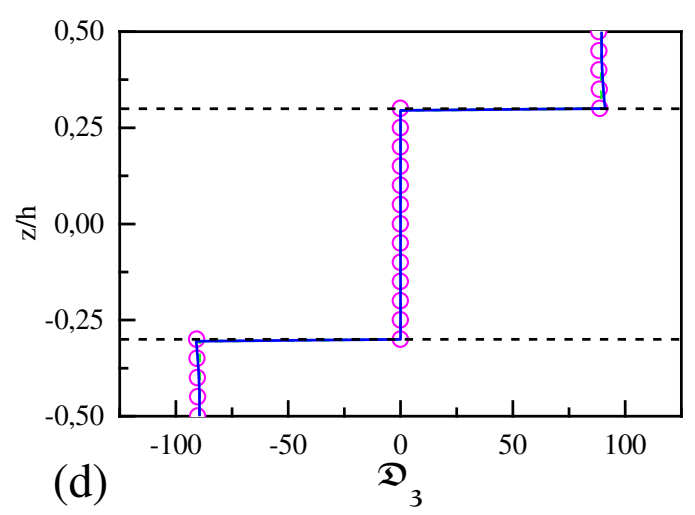

Fig.9 : Force density applied on the top face of a piezoelectric sandwich plate in closed circuit for $L / h=10$.

b - Applied electric potential.

The through-the-thickness profiles of electromechanical quantities produced by an applied 


\begin{tabular}{cccccccc}
\hline \hline L/h & Approaches & $\begin{array}{c}W \\
(L / 2,0)\end{array}$ & $\begin{array}{c}\text { Error } \\
\%\end{array}$ & $\begin{array}{c}\Phi \\
\left(L / 2,\left(h_{2}+h_{3}\right) / 2\right)\end{array}$ & $\begin{array}{c}\text { Error } \\
\%\end{array}$ & $\begin{array}{c}\llbracket T_{11} \rrbracket \\
\left(L / 2, z_{I}\right)\end{array}$ & $\begin{array}{c}\text { Error } \\
\%\end{array}$ \\
\hline \multirow{3}{*}{50} & F.E.M. & $-1.321 \times 10^{6}$ & & -2.807 & & 532.6 & \\
& Present & $-1.319 \times 10^{6}$ & 0.12 & -2.805 & 0.08 & 508.19 & 4.58 \\
& L.K. & $-1.312 \times 10^{6}$ & 0.6 & -2.793 & 0.48 & 508.15 & 4.6 \\
\hline \multirow{2}{*}{10} & F.E.M. & -2491.0 & & -0.1255 & & 20.73 & \\
& Present & -2372.97 & 4.74 & -0.1231 & 1.9 & 20.36 & 1.76 \\
& L.K. & -2100.14 & 15.7 & -0.1117 & 11 & 20.35 & 1.8 \\
\hline \hline
\end{tabular}

Table 7: Piezoelectric sandwich plate, applied density force.

electric potential are shown in Fig.10. The longitudinal displacement (see Fig.10.a) has a linear variation leading to a global bending deformation of the sandwich plate. In Fig.10.b the straight line corresponds to the deflection given by the present plate model. We note that the result provided by the simplified model is less accurate. The variation of the electric potential (see Fig.10.c) is well described by the expansions Eq.(51), the profile is linear in the piezoelectric layers while it is constant through the dielectric part. The variation of the longitudinal stress is shown in Fig.10.d, the profile exhibits very clearly the jump at the layer interfaces. The discrepancies between the present model, the simplified model and finite element simulations are presented in Table 8 for two slenderness ratios

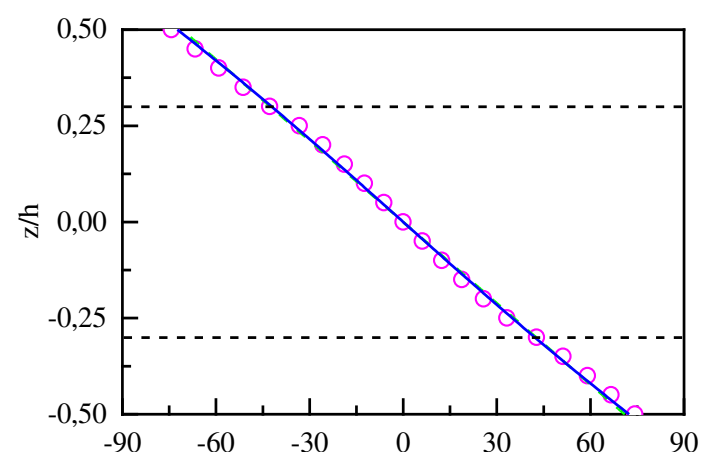

(a)

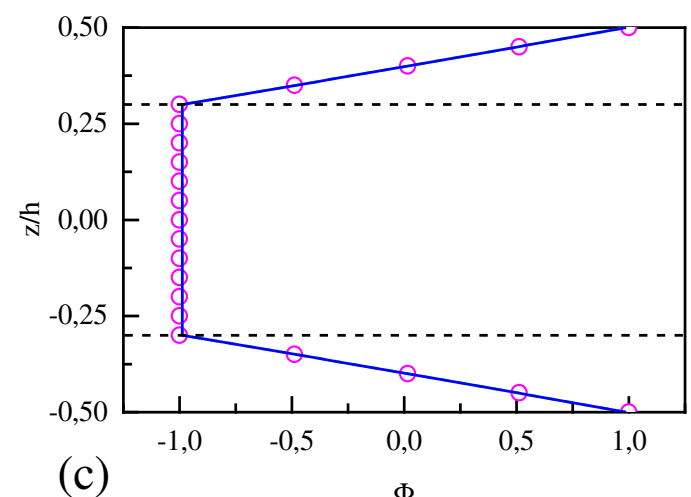

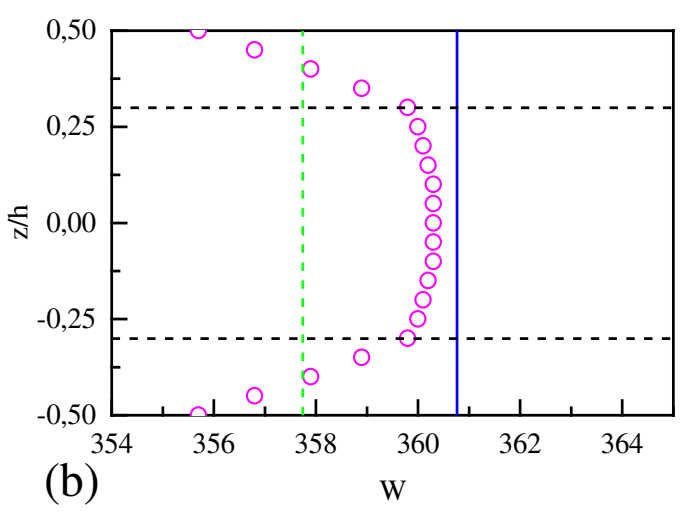

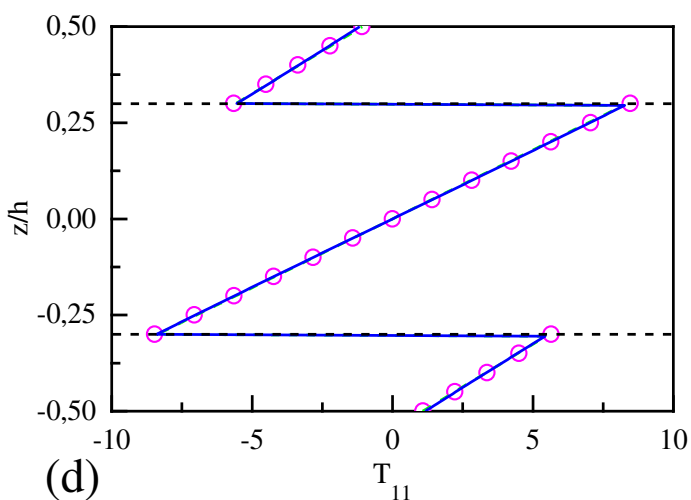

(d)

Fig.10 : Electric potential applied to a piezoelectric sandwich plate for $L / h=10$. 


\begin{tabular}{cccccccc}
\hline \hline L/h & Approaches & $\begin{array}{c}W \\
(L / 2,0)\end{array}$ & $\begin{array}{c}\text { Error } \\
\%\end{array}$ & $\begin{array}{c}U \\
(0, h / 2)\end{array}$ & $\begin{array}{c}\text { Error } \\
\%\end{array}$ & $\begin{array}{c}\llbracket \mathcal{D}_{3} \rrbracket \\
\left(L / 2, z_{I}\right)\end{array}$ & $\begin{array}{c}\text { Error } \\
\%\end{array}$ \\
\hline \multirow{4}{*}{50} & F.E.M. & 8947.0 & & -360.5 & & -112.70 & \\
& Present & 8946.509 & 0.005 & -355.5 & 1.38 & -111.345 & 1.20 \\
& L.K. & 8943.508 & 0.04 & -354.95 & 1.54 & -111.367 & 1.18 \\
\hline \multirow{4}{*}{10} & F.E.M. & 360.3 & & -74.3 & & -112.7 & \\
& Present & 360.76 & 0.13 & -72.38 & 2.57 & -111.356 & 1.2 \\
& L.K. & 357.74 & 0.71 & -71.0 & 4.44 & -111.385 & 1.17 \\
\hline \hline
\end{tabular}

Table 8: Piezoelectric sandwich plate, applied electric potential.

$L / h=10$ and $L / h=50$ for the maximum of the deflection at the plate center, the longitudinal displacement at the top face and the jump of the electric induction component $\mathcal{D}_{3}$ at the upper interface $\left(z=z_{I}\right)$. It is worthwhile noting that, once again, our approach leads to excellent results less than $1.4 \%$ for $L / h=50$. Although the simplified model provides rather correct predictions, it is less than the refined model. From the practical point of view, the piezoelectric sandwich $(L / h=50)$ produces a maximum deflection of the order of $90 \mu \mathrm{m}$ for an applied potential of 100 Volts. The surface density of the electric charge produced at the interface electrodes is given by the jump of the electric induction $\left.\llbracket D_{3}\right]_{z=z_{I}}=Q$ and we have $Q \approx 3 \times 10^{-2} \mathrm{C} / \mathrm{m}^{2}$. Such a structure is an excellent candidate to design performant actuators. This piezoelectric structure has been particularly studied leading to more or less similar predictions with various approaches among them we quote (Zhang and Sun, 1996; Benjeddou et al., 2000; Vinhas-Bertolini, 2001).

\section{Concluding remarks.}

A new refined approach to piezoelectric laminated plates is presented and analyzed in details displaying its performances and efficiency. The theory is mainly based on the principle of linear piezoelectricity in the framework of the quasi electrostatic hypothesis. It turns out that the present laminated plate model improves the performance of responses of piezoelectric components involved in smart structures. The model thus proposed seems to be a simple compromise between response accuracy and computational and analytical efficiency. More precisely the model is based on the combination of an equivalent singlelayer approach for the mechanical displacements with a layerwise-type modelling for the electric potential considered as an additional degree of freedom. Furthermore, the present approach accounts for the shearing effects, which plays a crucial role in the accuracy of the results. The layerwise modelling enables us a better description of the variations of electric field through-the-thickness of the laminated plate. Then such an approach can incorporate the local electromechanical response of each layer. Especially, the layerwise approach becomes a necessity when electric potentials are applied to electrodes at layer interfaces. This theory is particularly efficient for strong variations in piezoelectric and dielectric properties of the layers.

The refined piezoelectric plate modelling has been tested for simply supported laminated plates under cylindrical bending undergoing electromechanical loads (force density and electric potential). The complete set of governing equations for the piezoelectric compos- 
ites is derived from the Hamiltonian variational principle using expansions of the elastic displacement and electric potential as function of the thickness coordinate as defined by Eq.(11) along with the boundary conditions around the plate contour. The variational formulation accounts for the continuity conditions on the electric potential and normal component of the electric induction at the layer interfaces by employing Lagrange multipliers. A Fourier series solution to plate equations is then considered to compute the field variations through the plate thickness. A number of numerical examples is presented for piezoelectric structures of practical interest such as piezoelectric bimorph, bilayer and piezoelectric sandwich for three characteristic aspect ratios, namely $L / h=5,10$ and 50 . It is observed that the refined model leads to excellent predictions of the local variations of the electromechanical variables. The error in estimating the global responses is overall within a range of $1-2 \%$ with respect to results obtained from finite element method for the full 3D model. Nevertheless, the limitations of the present approach concerns the estimation of the transverse shear stress over the plate thickness. The shear stress cannot be estimated correctly (except for the piezoelectric bimorph) in the framework of the present model and a full layerwise approach should be required, which is envisaged in a further study. Furthermore, in view of the these results concerning sensor and actuator functions of piezoelectric laminated composites, we are strongly encouraged to extend the present study to the investigation of vibrations of laminated piezoelectric plates (Heyliger and Saravanos, 1995) yielding the concept of passive or active control of vibrations (Anderson and Hagood, 1994; Saravanos, 1999). This will be presented in a future work.

\section{Appendix A : Matrix form of constitutive equations for linear piezoelectricity.}

The constitutive equations (10) can be written in the following matrix form for materials possessing three mutually perpendicular planes of symmetry (orthotropic symmetry) (we use the Voigt notation with indices)

$$
\left[\begin{array}{c}
\sigma_{1} \\
\sigma_{2} \\
\sigma_{3} \\
\sigma_{4} \\
\sigma_{5} \\
\sigma_{6} \\
D_{1} \\
D_{2} \\
D_{3}
\end{array}\right]=\left[\begin{array}{ccccccccc}
C_{11}^{E} & C_{12}^{E} & C_{13}^{E} & 0 & 0 & 0 & 0 & 0 & -e_{31} \\
C_{12}^{E} & C_{22}^{E} & C_{23}^{E} & 0 & 0 & 0 & 0 & 0 & -e_{32} \\
C_{13}^{E} & C_{23}^{E} & C_{33}^{E} & 0 & 0 & 0 & 0 & 0 & -e_{33} \\
0 & 0 & 0 & C_{44}^{E} & 0 & 0 & 0 & -e_{24} & 0 \\
0 & 0 & 0 & 0 & C_{55}^{E} & 0 & -e_{15} & 0 & 0 \\
0 & 0 & 0 & 0 & 0 & C_{66}^{E} & 0 & 0 & 0 \\
0 & 0 & 0 & 0 & e_{15} & 0 & \epsilon_{11}^{S} & 0 & 0 \\
0 & 0 & 0 & e_{24} & 0 & 0 & 0 & \epsilon_{22}^{S} & 0 \\
e_{31} & e_{32} & e_{33} & 0 & 0 & 0 & 0 & 0 & \epsilon_{33}^{S}
\end{array}\right]\left[\begin{array}{c}
S_{1} \\
S_{2} \\
S_{3} \\
S_{4} \\
S_{5} \\
S_{6} \\
E_{1} \\
E_{2} \\
E_{3}
\end{array}\right] .
$$

If the material system has an axis of symmetry (transversally isotropic) the number of independant material coefficients is reduced according to $C_{11}^{E}=C_{22}^{E}, C_{13}^{E}=C_{23}^{E}, C_{44}^{E}=C_{55}^{E}$, $C_{66}^{E}=\frac{1}{2}\left(C_{11}^{E}-C_{12}^{E}\right), e_{31}=e_{32}, e_{15}=e_{24}$ and $\epsilon_{11}^{S}=\epsilon_{22}^{S}$.

\section{Appendix B : Coefficients of Lagrange multipliers.}


The coefficients of the arbitrary variations in unknown functions introduced in the virtual work associated with Lagrange multipliers (see Eq.(27)) are computed from continuity conditions Eq.(15) using Eq.(16) and the approximations defined by Eq.(11) starting from Eq.(26). They depend on the Lagrange multipliers and can be put in the following form

$$
\begin{aligned}
A_{\alpha \beta}^{u} & =\sum_{\ell=1}^{N-1}\left(e_{3 \alpha \beta}^{*(\ell+1)}-e_{3 \alpha \beta}^{*(\ell)}\right) \nu_{\bar{e}(\ell)}, \\
A_{\alpha \beta}^{w} & =\sum_{\ell=1}^{N-1} z_{I}^{(\ell)}\left(e_{3 \alpha \beta}^{*(\ell+1)}-e_{3 \alpha \beta}^{*(\ell)}\right) \nu_{\bar{e}(\ell)}, \\
A_{\alpha \beta}^{\gamma} & =\sum_{\ell=1}^{N-1} f\left(z_{I}^{(\ell)}\right)\left(e_{3 \alpha \beta}^{*(\ell+1)}-e_{3 \alpha \beta}^{*(\ell)}\right) \nu_{\bar{e}(\ell)}, \\
A_{0}^{+(\ell)} & =\mu_{\ell}, \\
A_{0}^{-(\ell)} & =-\hat{\mu}_{\ell}, \\
A_{1}^{+(\ell)} & =-\left(\frac{1}{2} h_{\ell+1} \mu_{\ell}+\epsilon_{33}^{*(\ell+1)} \nu_{\bar{e}(\ell)}\right), \\
A_{1}^{-(\ell)} & =-\left(\frac{1}{2} h_{\ell} \hat{\mu}_{\ell}-\epsilon_{33}^{*(\ell)} \nu_{\bar{e}(\ell)}\right), \\
A_{2}^{+(\ell)} & =h_{\ell+1} \epsilon_{33}^{*(\ell+1)} \nu_{\bar{e}(\ell)}, \\
A_{2}^{-(\ell)} & =h_{\ell} \epsilon_{33}^{*(\ell)} \nu_{\bar{e}(\ell)}, \\
A_{3}^{+(\ell)} & =g\left(z_{I}^{(\ell)}\right) \mu_{\ell}+\frac{\pi}{h} f\left(z_{I}^{(\ell)}\right) \epsilon_{33}^{*(\ell+1)} \nu_{\bar{e}(\ell),}, \\
A_{3}^{-(\ell)} & =-g\left(z_{I}^{(\ell)}\right) \hat{\mu}_{\ell}-\frac{\pi}{h} f\left(z_{I}^{(\ell)}\right) \epsilon_{33}^{*(\ell)} \nu_{\bar{e}(\ell)},
\end{aligned}
$$

where the effective piezoelectric and dielectric constants of the $\ell$ th layer $e_{3 \alpha \beta}^{*(\ell)}$ and $\epsilon_{33}^{*(\ell)}$ are given in Appendix E. We have set $\hat{\mu}_{\ell}=\mu_{\ell}-\rho_{e(\ell)}$.

\section{Appendix C : Matrix components of constitutive relations.}

The present appendix provides one with the components of the matrices introduced in Section 6 in the constitutive equations for the generalized resultants of the plate model (Voigt notation has been considered).

- The matrices $\mathbb{A}, \mathbb{B}, \mathbb{D}, \mathbb{E}, \hat{\mathbb{E}}$ and $\hat{\mathbb{D}}$ are $3 \times 3$ matrices defined as follows

$$
(\mathbb{A}, \mathbb{B}, \mathbb{D}, \mathbb{E}, \hat{\mathbb{E}}, \hat{\mathbb{D}})=\sum_{\ell=1}^{N}\left(a_{\ell}, b_{\ell}, c_{\ell}, \alpha_{\ell}, \beta_{\ell}, \zeta_{\ell}\right)\left[\begin{array}{ccc}
C_{11}^{*(\ell)} & C_{12}^{*(\ell)} & 0 \\
C_{12}^{*(\ell)} & C_{22}^{*(\ell)} & 0 \\
0 & 0 & C_{66}^{*(\ell)}
\end{array}\right]
$$

- $\mathbb{P}_{k}=\left[\mathbb{P}_{k}^{(1)}, \cdots, \mathbb{P}_{k}^{(N)}\right] k \in\{1,2,3\}$ is a $3 \times 3 N$ matrix made of $N$ blocks of $3 \times 3$ 
matrices $\mathbb{P}_{k}^{(\ell)}$ given by

$$
\begin{gathered}
\left(\mathbb{P}_{1}^{(\ell)}, \mathbb{P}_{2}^{(\ell)}, \mathbb{P}_{3}^{(\ell)}\right)= \\
{\left[e_{31}^{*(\ell)}, e_{32}^{*(\ell)}, 0\right]^{T} \times\left(\left[a_{\ell}, 0,-\frac{\pi}{h} \alpha_{\ell}\right],\left[b_{\ell},-d_{\ell},-\frac{\pi}{h} \beta_{\ell}\right],\left[\alpha_{\ell},-\frac{\pi}{h} \delta_{\ell},-\frac{\pi}{h} \zeta_{\ell}\right]\right),}
\end{gathered}
$$

- $\mathbb{H}$ is a $3 N \times 3 N$ matrix made of $N$ blocks of $3 \times 3$ matrices in its diagonal

$$
\mathbb{H}=\left[\begin{array}{ccc}
\mathbb{H}^{(1)} & & \\
& \ddots & \\
& & \mathbb{H}^{(N)}
\end{array}\right] \text { with } \mathbb{H}^{(\ell)}=\epsilon_{33}^{*(\ell)}\left[\begin{array}{ccc}
-a_{\ell} & 0 & \frac{\pi}{h} \alpha_{\ell} \\
0 & 2 d_{\ell} & -\frac{\pi^{2}}{h^{2}} \delta_{\ell} \\
\frac{\pi}{h} \alpha_{\ell} & -\frac{\pi^{2}}{h^{2}} \delta_{\ell} & -\frac{\pi^{2}}{h^{2}} \zeta_{\ell}
\end{array}\right],
$$

- $\mathbb{R}$ is a $2 \times 2$ matrix defined by

$$
\mathbb{R}=\sum_{\ell=1}^{N} \frac{\pi^{2}}{h^{2}} \bar{\zeta}_{\ell}\left[\begin{array}{cc}
C_{55}^{*(\ell)} & 0 \\
0 & C_{44}^{*(\ell)}
\end{array}\right],
$$

- $\mathbb{L}_{\alpha}$ is a matrix made of $N$ blocks of $2 \times 4$ matrices given by

$$
\mathbb{L}_{\alpha}=\left[\mathbb{L}_{\alpha}^{(1)}, \cdots, \mathbb{L}_{\alpha}^{(N)}\right] \quad \alpha \in\{1,2\},
$$

with

$$
\left(\mathbb{L}_{1}^{(\ell)}, \mathbb{L}_{2}^{(\ell)}\right)=\frac{\pi}{h}\left(\left[e_{15}^{*(\ell)}, 0\right]^{T},\left[0, e_{24}^{*(\ell)}\right]^{T}\right) \times\left[\bar{\alpha}_{\ell}, \frac{\pi}{2 h} \bar{\delta}_{\ell}, \delta_{\ell}, \bar{\zeta}_{\ell}\right]
$$

- $\mathbb{T}_{\alpha}$ is a $4 N \times 4 N$ matrix consisting of $N$ blocks of $4 \times 4$ matrices in its diagonal

$$
\mathbb{T}_{\alpha}=\left[\begin{array}{ccc}
\mathbb{T}_{\alpha}^{(1)} & & \\
& \ddots & \\
& & \mathbb{T}_{\alpha}^{(N)}
\end{array}\right] \text { with } \mathbb{T}_{\alpha}^{(\ell)}=-\epsilon_{\alpha \alpha}^{*(\ell)}\left[\begin{array}{cccc}
a_{\ell} & 0 & d_{\ell} & \bar{\alpha}_{\ell} \\
0 & -\frac{d_{\ell}}{2} & 0 & \frac{\pi}{2 h} \bar{\delta}_{\ell} \\
d_{\ell} & 0 & f_{\ell} & \delta_{\ell} \\
\bar{\alpha}_{\ell} & \frac{\pi}{2 h} \bar{\delta}_{\ell} & \delta_{\ell} & \bar{\zeta}_{\ell}
\end{array}\right]
$$

Remark. For transversally isotropic material the above matrices can be reduced due to material symmetry. Then, in this case, in the block $\mathbb{P}_{k}^{(\ell)}$ we have $e_{31}^{*(\ell)}=e_{32}^{*(\ell)}$. The matrix $\mathbb{R}$ is a diagonal matrix with $C_{44}^{*(\ell)}=C_{55}^{*(\ell)}$. In addition, we have $\left(\mathbb{L}_{1}\right)_{1} \neq 0,\left(\mathbb{L}_{1}\right)_{2}=0$, $\left(\mathbb{L}_{2}\right)_{1}=0$ and $\left(\mathbb{L}_{2}\right)_{2} \neq 0$. At last, since $\epsilon_{11}^{*(\ell)}=\epsilon_{22}^{*(\ell)}$, this yields $\mathbb{T}_{1}=\mathbb{T}_{2}$. 


\section{Appendix D : Coefficients of the matrix components.}

The different coefficients introduced in the definitions of the matrix components (see Appendix C) are only functions of the layer thickness and interface coordinates.

$$
\left.\begin{array}{l}
a_{\ell}=z_{I}^{(\ell)}-z_{I}^{(\ell-1)}, \\
b_{\ell}=\frac{1}{2}\left[\left(z_{I}^{(\ell)}\right)^{2}-\left(z_{I}^{(\ell-1)}\right)^{2}\right], \\
c_{\ell}=\frac{1}{3}\left[\left(z_{I}^{(\ell)}\right)^{3}-\left(z_{I}^{(\ell-1)}\right)^{3}\right], \\
d_{\ell}=-\frac{1}{6}\left[z_{I}^{(\ell)}-z_{I}^{(\ell-1)}\right]^{3}, \\
f_{\ell}=\frac{1}{30}\left[z_{I}^{(\ell)}-z_{I}^{(\ell-1)}\right]^{5}, \\
\alpha_{\ell}=-\frac{h}{\pi}\left[g\left(z_{I}^{(\ell)}\right)-g\left(z_{I}^{(\ell-1)}\right)\right], \\
\beta_{\ell}=\frac{h}{\pi}\left\{\frac{h}{\pi}\left[f\left(z_{I}^{(\ell)}\right)-f\left(z_{I}^{(\ell-1)}\right)\right]-\left[z_{I}^{(\ell)} g\left(z_{I}^{(\ell)}\right)-z_{I}^{(\ell-1)} g\left(z_{I}^{(\ell-1)}\right)\right]\right\}, \\
\delta_{\ell}=-\frac{h^{2}}{\pi^{2}}\left\{\frac{2 h}{\pi}\left[f\left(z_{I}^{(\ell)}\right)-f\left(z_{I}^{(\ell-1)}\right)\right]-\left(z_{I}^{(\ell)}-z_{I}^{(\ell-1)}\right)\left[g\left(z_{I}^{(\ell)}\right)+g\left(z_{I}^{(\ell-1)}\right)\right]\right\}, \\
\zeta_{\ell}=\frac{h^{2}}{2 \pi^{2}}\left\{\left[z_{I}^{(\ell)}-z_{I}^{(\ell-1)}\right]-\frac{\pi}{h}\left[g\left(z_{I}^{(\ell)}\right) f\left(z_{I}^{(\ell)}\right)-g\left(z_{I}^{(\ell-1)}\right) f\left(z_{I}^{(\ell-1)}\right)\right]\right\}, \\
\bar{\alpha}_{\ell}=\frac{h}{\pi}\left[f\left(z_{I}^{(\ell)}\right)-f\left(z_{I}^{(\ell-1)}\right)\right], \\
\bar{\delta}_{\ell}=\frac{h^{2}}{\pi^{2}}\left\{\frac{2 h}{\pi}\left[g\left(z_{I}^{(\ell)}\right)-g\left(z_{I}^{(\ell-1)}\right)\right]+\left(z_{I}^{(\ell)}-z_{I}^{(\ell-1)}\right)\left[f\left(z_{I}^{(\ell)}\right)+f\left(z_{I}^{(\ell-1)}\right)\right]\right\}, \\
\bar{\zeta}_{\ell}=\frac{h^{2}}{2 \pi^{2}}\left\{\left[z_{I}^{(\ell)}-z_{I}^{(\ell-1)}\right]+\frac{\pi}{h}\left[g\left(z_{I}^{(\ell)}\right) f\left(z_{I}^{(\ell)}\right)-g\left(z_{I}^{(\ell-1)}\right) f\left(z_{I}^{(\ell-1)}\right)\right]\right\} .
\end{array}\right\},
$$

\section{Appendix E : Effective elastic, piezoelectric and dielectric constants.}

The effective modulus of elasticity due to the normal shear stress hypothesis for elastic thin plates $\left(\sigma_{33}\right.$ negligeable in comparison to the other stress components, it is of $(h / L)^{3}$ order) are given by

$$
C_{a b}^{*}=C_{a b}^{E}-\frac{C_{a 3}^{E} C_{3 b}^{E}}{C_{33}^{E}} .
$$

On using the same argument, we have the effective piezoelectric coefficients

$$
e_{j a}^{*}=e_{j a}-\frac{e_{j 3} C_{a 3}^{E}}{C_{33}^{E}},
$$

and the effective dielectric constants

$$
\epsilon_{i j}^{*}=\epsilon_{i j}^{S}+\frac{e_{i 3} e_{j 3}}{C_{33}^{E}}
$$

respectively, with $a, b \in\{1, \cdots, 6\}, \quad i, j \in\{1,2,3\}$ when using the Voigt notation for convenience. 


\section{References.}

Anderson E.H., Hagood N.W., 1994. Simultaneous piezoelectric sensing/actuation : Analysis and application to controlled structures. J. Sound and Vibration 74, 617-639.

Benjeddou A., Trindade M.A., Ohayon R., 2000. Piezoelectric actuation mechamism for intelligent sandwich structures. Smart Mater. Struct. 9, 328-335.

Bisegna P., Maceri F., 1996. An exact three-dimensional solution for simply supported rectangular piezoelectric plates. J. Appl. Mech. 63, 628-638.

Carrera E., 1997. An improved Reissner-Mindlin-type model for the electromechanical analysis of multilayered plates including piezo-layers. J. Intelligent Mat. Syst. and Struct. 8, 232-248.

Chandrashekhara K., Donthireddy P., 1997. Vibration suppression of composite beams with piezoelectric devices using a higher order theory. Eur. J. Mech. A/Solids 16, 709-721.

Cheng Z-Q, Batra R.C., 2000. Three-dimensional asymptotic analysis of multiple-electroded piezoelectric laminates. AIAA Journal 38, 317-324.

Cheng Z-Q., Lim C.W., Kitipornchai S., 2000. Three-dimensional asymptotic approach to inhomogeneous and laminated piezoelectric plates. Int. J. Solids Struct. 37, 3153-3175.

Fernandes A., 2000. Modèle et étude de composants piézoélectriques : Applications aux structures multifonctionnelles. Thesis, University Pierre et Marie Curie, Paris.

Fernandes A., Pouget J., 2001-a. Two-dimensional modelling of laminated piezoelectric composites : Analysis and numerical results. J. Thin-Walled Struct. 39, 3-22.

Fernandes A., Pouget J., 2001-b. An accurate modelling of piezoelectric plates. Single-layered plate. Arch. Appl. Mech. 71, 509-524 (2001).

Gopinathan S.V., Varadan V.V., Varadan V.K., 2000. A review and critique of theories for piezoeletric laminates. Smart Mater. Struct. 9, 24-48.

Hagood N.W., Von Flotow A., 1991. Damping of structural vibrations with piezoelectric materials and passive electrical networks. J. Sound and Vibration 146, 243-268.

Heyliger P., Saravanos D.A., 1995. Exact free vibration analysis of laminated plates with embedded piezoelectric layers. J. Acoust. Soc. Am. 98, 1547-1557.

Heyliger P., Brook S., 1996. Exact solution for laminated piezoelectric plates in cylindrical bending. J. Appl. Mech. 63, 903-910.

Icardi U., Di Scuva M., 1996. Large-deflection and strain analysis of multilayered plates with induced-strain actuators. Smart Marter. Struct. 5, 140-164.

Ikeda T., 1996. Fundamental of piezoelectricity (Oxford University Press, Oxford).

Kim J., Varadan V.V., Varadan V.K., 1997. Finite element modelling of structures including piezoelectric active devices. Int. J. Num. Meth. Engng. 40, 817-832.

Lee C.K., Moon F.C., 1989. Laminated piezopolymer plates for torsion, bending sensors, actuators. J. Acoust. Soc. Am. 85, 2432-2439.

Lee C.K., 1990. Theory of laminated piezoelectric plates for the design of distributed sensors/actuators.

Part 1 : Governing equations and reciprocal relationships. J. Acoust. Soc. Am. 87, 1144-1158.

Loewy R.G., 1997. Recent developments in smart structures with aeronautical applications. Smart Mater. Struct. 6, R11-R42.

Maugin G.A., 1985. Nonlinear electromechanical effects and applications, World Scientific, Singapore.

Maugin G.A., Attou D., 1990. An asymptotic theory of thin piezoelectric plates. Q. J. Mech. Appl. Math. 43, 347-362.

Mindlin R.D., 1972. High frequency vibrations of piezoelectric crystal plates. Int. J. Solids Struct. 8, 895-906.

Mitchell J.A., Reddy J.N., 1995. A refined plate theory for composite laminates with piezoelectric laminae. Int. J. Solids Struct. 32, 2345-2367. 
Muralt P., Dohl D.W., Denk W., 1986. Wide-range, low-operating-voltage, bimorph stm : Application as potentiometer. IBM J. Res. Develop. 30, 443-450.

Ochoa O.O., Reddy J.N., 1992. Mechanics of composite laminates. In Finite Element Analysis of Composite Laminates, Solid Mechanics and its Applications, vol.7, Klumer Academic Publishers, Dordrecht, pp.5-36.

Pai P.F., Nayfeh A.H., Oh K., Mook D.T., 1993. A refined nonlinear model of composite plates with integrated piezoelectric actuators and sensors. Int. J. Solids Struct. 30, 1603-1630.

Rao S.S., Sunar M., 1994. piezoelectricity and its use in disturbance sensing and control of flexible structures : A survey. Appl. Mech. Rev. 47, 113-123.

Reddy J.N., 1984. A simple higher-order theory for laminated composite plates. J. Appl. Mech., 745-752.

Robbins D.H., Reddy J.N., 1993. Modelling of thick composites using a layerwise laminate theory. Int. J. Num. Methods Engng. 36, 655-677.

Saravanos D.A., Heyliger P.R., Hopkins D.A., 1997. Layerwise mechanics and finite element for the dynamic analysis of piezoelectric composite plates. Int. J. Solids Struct. 34, 359-378.

Saravanos D.A., 1999. Damped vibration of composite plates with passive piezoelectric-resistor elements. J. Sound and Vibration 221, 867-885.

Saravanos D.A., Heyliger P.R., 1999. Mechanics and computational models for laminated piezoelectric beams, plates and shell. Appl. Mech. Rev. 52, 305-320.

Smits J.G., Dalke S.I., Cooney T.K., 1991. The constituent equations of piezoelectric bimorphs. Sensors and Actuators A28, 41-61.

Sunar M., Rao S.S., 1999. Recent advances in sensing and control of flexible structures via piezoelectric materials technology. Appl. Mech. Rev. 52, 1-16.

Tani J., Takagi T., Qiu J., 1998. Intelligent material systems : Application of functional material. Appl. Mech. Rev. 51, 505-521.

Tiersten H.F., 1969. Linear piezoelectric plate vibrations, Plenum, New York.

Touratier M., 1991. An efficient standard plate theory. Int. J. Engng. Sci. 29, 901-916.

Tzou H.S., 1993. Piezoelectric shells : distributed sensing and control of continua, Klumer Academic Publisher, Dordrecht.

Vinhas-Bertolini, 2001. Modélisation des poutres sandwich élasto-piézo-électriques : Eléments finis raffinés. Thesis, Ecole Nationale Supérieure d'Art et Métier, Paris.

Wang B., Rogers C.A., 1991. Laminated plate theory for spatially distributed induced strain actuators. J. Composite Mat. 25, 433-452.

Wang J., Yong Y-K., Imai T., 1999. Finite element analysis of the piezoelectric vibration of quartz plate resonators with higher-order plate theory. Int. J. Solids Struct. 36, 2303-2319.

Wang J., Yang J., 2000. Higher-order theories of piezoelectric plates and applications. J. Appl. Mech. Rev. 53, 87-99.

Yang J.S., 1999. Equation for thick elastic plates with partially electroded piezoelectric actuators and higher order electric fields. Smart Mater. Struct. 8, 73-82.

Yoon H.S., Washington G., 1998. Piezoceramic actuated aperture antennae. Smart Mater. Struct. 7, 537-542.

Zhang X.D., Sun C.T., 1996. Formulation of an adaptive sandwich beam. Smart Mater. Struct. 5, 814-823. 